\title{
A Spatially Variant Rebalancing Method for Discrete-Ordinates Transport Equation
}

\author{
Wesley Ford, Emiliano Masiello, \\ Christophe Calvin, François Fevotte, Bruno Lathuilliere
}

November 20, 2018

\begin{abstract}
In this paper we propose a new non-linear technique for accelerating source iterations for the discrete-ordinates transport equation. The method, called Spatially Variant Rebalancing Method (SVRM), is based on the computation of the zero and first order spatial variation of the neutron balance equation. The non-linear acceleration is applied to the method of characteristics (MOC) with a step-approximation of the source. The new acceleration is meant to catch high-order variation of the neutron flux within the spatial mesh. The paper proposes a numerical analysis of the technique based on the explicit computation of the Jacobian. The latter is analyzed with both spectral and Fourier analysis. Also, a comparison of the new method with CMFD, DSA, and BPA has been done for a parametrized heterogeneous problem that allows the study of the performances of the method in different transport regimes.
\end{abstract}

\section{Introduction}

The time to solution of large-scale deterministic neutron transport codes are often based on the synergy of the source iteration operator (SI operator, also referred to as the transport operator) and the acceleration operator. While the transport operator defines the accuracy of the results, the acceleration operator speeds up the convergence of the SI. This acceleration is typically achieved by converging the scattering and of the fission source. The SI operator alone converges in a reasonable time only when the problem is dominated by absorption and high rates of leakage. Contrarily, for the simulation of a nuclear reactor, the SI shows poor performances since the transport regime is dominated by scattering [1]. Various forms of coarse-mesh rebalancing methods (CMR) were proposed in the early days of transport simulations as an acceleration methodology [2]. These non-linear methods were improved by the coarse-mesh finite differences method (CMFD) that was developed independently later on 3 . 
The CMFD method has proved effective in various successful numerical implementations, especially in synergy with the method of characteristics (MOC) SI operator [3]. The non-linear nature of the acceleration has allowed it to effectively reduce the number of transport iterations while reducing the memory footprint. However, despite these beneficial attributes, CMFD can lead to unstable or divergent iteration behaviour for some critical spatial configurations. Cho and Park have shown that the CMFD is effective when the coarse cell optical thickness does not exceed 1 mean free path (mfp), but CMFD becomes rapidly unstable for cells with large optical thicknesses [4]. One of the aspects of CMFD that causes this instability is it's representation of the spatial variation of flux not conforming to that of transport. For example, in the case of MOC, even though the source has a step approximation, the angular flux and, thus, the scalar flux have an exponential variation within the cell. However, The spatial distribution of the flux within the cell is not commonly provided by the transport operator. In the case of the standard step approximation of the MOC, only the average scalar flux (or, more generally the average angular moments) is computed/provided, for the purpose of preserving consistency of the source. But the contribution of the high order variation of the flux is implicitly contained by the flux on the boundary of the cell thanks to the 'exact' propagation equation. Moreover, in the case that a coarse model of the transport mesh is used by CMFD, the variation of the flux within the coarse cell is not taken into account do to the lack of available degrees of freedom. Thus, taking advantage of the high order flux variation contained within the interface flux and increasing the number of spatial degrees of freedom are the principal motivations behind the nonlinear rebalancing method presented in this article.

In this paper, a spatially variant rebalancing method (SVRM) is proposed. This method takes into account the linear spatial variation of the flux within the cell and has been designed to offer better stability and performance than the currently available acceleration methods. It is meant to accomplish this by using a higher order set of equations to represent the scalar flux and surface current relationship within a spatial region. This is particularly necessary when the neutron flux is convective within the cell. While, when the neutron flux is diffusive, the method reduces to a CMR method. In this paper we will discuss the derivation of the SVRM method and analyze the results of it's spectral radius, Fourier stability analysis, and numeric benchmarking in 1D. The 1D transport equation is solved by means of the MOC equations with a step approximation.

The SVRM method is based on two balance equations, both obtained by the original transport equation. The first is the classical zero-order balance equation which is utilized by the most common rebalancing methods such as CMR or CMFD, while the second is the first order balance equation that takes into account the linear variation of the flux. This second equation is obtained by typical projection technique of the particle balance equation onto the linear space. In particular, the weight functions are Legendre polynomials up to the first order while the trial function is parabolic. The SVRM balance equations are completed by four closures equations relating the average flux, the 
interface flux and the average current to the interface current. Compared to CMFD, SVRM has an additional balance equation that doubles the number of degrees of freedom. In the formulation that we propose, instead of solving for the flux, as is done by CMFD and CMR, SVRM solves for the partial outgoing currents. This choice has been made to allow for the correction of the surface interface-flux, speeding up the convergence of the incoming angular flux. This correction is particularly effective when the transport operator is solved using a Block-Jacobi iteration scheme, as is commonly done for parallel schemes based on domain decomposition.

The SVRM method has been particularly addressed to speed up the convergence of parallel computing. In this paper, the Gauss-Jacobi transport iteration will be analyzed with particular attention. In this particular iteration scheme, each region is potentially solved independently from the others. Moreover, all the problems we propose deals with homogeneous boundary conditions. Also in this case, the upgrade of the boundary angular flux is done by a Gauss-Jacobi iteration (instead of a direct elimination.) As we will see in the spectral analysis, the boundary conditions upgrade particularly affects the convergence of optically thin problems, when the convergence of the boundary flux is dominant with respect to the iterior flux.

The rest of paper is organized as follows: Section 2 explains the SVRM in terms of balances equations, closure relations and consistency, Section 3 shows the transport operator and matrix formalism that we adopted for the numerical analysis out of convenience. Then, Section 4 is dedicated to the computation of the Jacobian and to the spectral and Fourier analysis, Section 6 depicts some numerical results and Section 7 is dedicated to the conclusions and further remarks.

\section{Spatially Variant Rebalance Method}

The first equation of SVRM is simply the neutron balance equation defined by Eq. (1) below for a discrete portion of space $x \in[-\Delta / 2, \Delta / 2]$.

$$
J(\Delta / 2)-J(-\Delta / 2)+\int_{-\Delta / 2}^{\Delta / 2} \Sigma_{r}(x) \phi(x) d x=\int_{-\Delta / 2}^{\Delta / 2} q(x) d x
$$

where $\phi(x)=\frac{1}{2} \int_{-1}^{1} \psi(x, \mu) d \mu$ is the scalar flux and $J(x)=\frac{1}{2} \int_{-1}^{1} \mu \psi(x, \mu) d \mu$ is the current, i.e. the zero and the first angular moments respectively, while $\Sigma_{r}(x)=\Sigma_{t}(x)-\Sigma_{s 0}(x)$ is the removal cross section while $x_{R}$ and $x_{L}$ are the right and left coordinates of the slab.

To obtain the second equation that characterizes the acceleration method, we introduce a first order spatial projection of the balance equation. After angular integration, we project the neutron balance equation on the linear subspace, by 
applying the integration $\int_{-\Delta / 2}^{\Delta / 2} x d x$ over the balance equation, obtaining

$$
\frac{J\left(x_{R}\right)+J\left(x_{L}\right)}{2} \Delta-\int_{x_{L}}^{x_{R}} J(x) d x+\int_{-\Delta / 2}^{\Delta / 2} \Sigma_{r}(x) \phi(x) x d x=\int_{-\Delta / 2}^{\Delta / 2} q(x) x d x .
$$

Eq. (2) represents the "balance" for the first-order spatial variation. The equations (1) and (2) are discretized to make the system numerically solvable. The effectiveness of the acceleration method is tied to how accurately the acceleration solution basis represents that of transport. In the classical rebalancing methods Eq. 22 reduces to the identity $\frac{1}{\left(x_{R}-x_{L}\right)} \int_{x_{L}}^{x_{R}} J(x) d x=\frac{J\left(x_{R}\right)+J\left(x_{L}\right)}{2}$, i.e. the average net current within the slab $\left[x_{L}, x_{R}\right]$ is equal to the linear average of the values of the current to the left and to the right, because the spatial representation of the flux and of the source is step constant. In order to solve the first order expansion, knowledge of the spatial distribution of the scalar flux and fixed source within a region is required. For example, in the step approximation of the source, the first order moment of the scalar flux in an homogeneous slab $\Delta$ can be explicitly computed by

$$
\begin{aligned}
\int_{-\Delta / 2}^{-\Delta / 2} d x x \phi(x)= & \int_{-\Delta / 2}^{\Delta / 2} d x x \int_{0}^{1} d \mu\left[\psi_{L}(\mu)-\frac{\Sigma_{s 0} \bar{\phi}+\bar{q}}{\Sigma}\right]\left(1-e^{-\Sigma(x+\Delta / 2) /|\mu|}\right)(3) \\
& +\int_{-\Delta / 2}^{\Delta / 2} d x x \int_{0}^{1} d \mu\left[\psi_{R}(-\mu)-\frac{\Sigma_{s 0} \bar{\phi}+\bar{q}}{\Sigma}\right]\left(1-e^{-\Sigma(\Delta / 2-x) /|\mu|}\right)
\end{aligned}
$$

Where $\psi_{L}(\mu)$ and $\psi_{R}(-\mu)$ are the left and right boundary angular fluxes, respectively, while $\bar{q}$ is the constant source. Such spatial moment equation is not provided by the standard MOC, which is based on the step approximation of the source. Because of such lack of knowledge of the spatial moments, the Eq. (2) must be artificially satisfied. Moreover, Eq. (2) becomes non-trivial when the flux and the source have a spatial variation within the discrete region. One of the goals of SVRM is to use a more accurate spatial representation by taking profit of valuable information contained in the interface quantities.

\subsection{Flux, source and current spatial representation}

Letting the function $f(x)$ represents the scalar flux or the current, the MOC provides in $x \in[-\Delta / 2, \Delta / 2]$ three values $\left(\bar{f}, f_{L}, f_{R}\right)$, which are the average of $f(x)$, and the point-wise values on the left and right boundaries, respectively. In this work, a parabolic trial function is used to represent the spatial distribution of $f(x)$ within each region. The generic function $f(x)$ is approximated by the parabolic function $\widetilde{f}(x)$, as depicted in Fig. 2.1.

$$
\widetilde{f}(x)=\bar{f}+\left(f_{R}-f_{L}\right) \frac{x}{\Delta}+4\left(\frac{f_{R}+f_{L}}{2}-\bar{f}\right)\left(3 \frac{x^{2}}{\Delta^{2}}-1\right) .
$$

The expansion (4) is constructed such that the following three conditions are met, 


$$
\begin{aligned}
\tilde{f}(-\Delta / 2) & =f_{L}, \\
\int_{-\Delta / 2}^{\Delta / 2} \widetilde{f}(x) d x & =\Delta \bar{f}, \\
\widetilde{f}(\Delta / 2) & =f_{R} .
\end{aligned}
$$

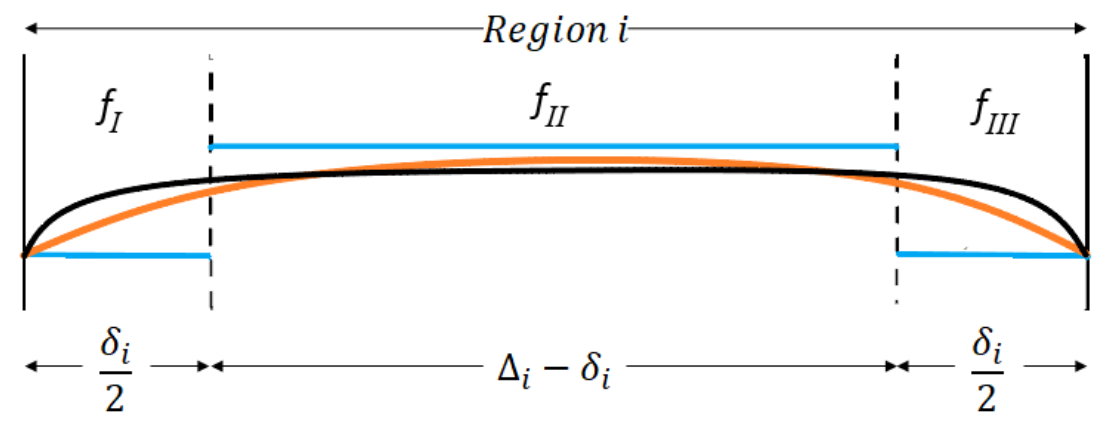

Figure 1: shows the spatial distribution of the scalar flux and fixed source within each spatial region, assumed by SVRM

Rewriting equations (1) and (2) using the parabolic trial function in the flux and current integrals, we obtain

$$
J_{R}-J_{L}+\tau_{r} \bar{\phi}=\bar{q} \Delta
$$

for Eq. (1) and

$$
\Delta\left(\frac{J_{R}+J_{L}}{2}-\bar{J}\right)+\widetilde{\delta} \tau_{r}\left(\phi_{R}-\phi_{L}\right)=\Delta \widetilde{\delta}\left(q_{R}-q_{L}\right)
$$

for Eq. (2). Here, the symbols are

- $\phi_{L / R}$ and $\bar{\phi}$ indicating respectively the interface scalar on the left/right side and the average scalar flux,

- $J_{L / R}$ and $\bar{J}$ indicating respectively the interface current on the left/right side and the average current,

- $q_{R / R}$ and $\bar{\phi}$ indicating respectively the interface scalar on the left/right side and the average scalar flux,

$\tau_{i, R}=\left(\Sigma_{t}-\Sigma_{s 0}\right) \Delta$ indicating the removal optical thickness, and 
- $\widetilde{\delta}$ is the modified spatial parameter that takes into account the first order variation, in particular

$$
\widetilde{\delta}=\frac{\Delta}{12}
$$

The parameter $\widetilde{\delta}$ plays a key role to discriminate the trial expansion basis'. By introducing a partition of the slab with the region index $i=1, \ldots, I$, where $I$ is the number of regions, and specializing Eqs. (6) and (7) in each spatial mesh, the acceleration method has 6 unknowns per mesh cell $i$,

$$
\left[\phi_{i, L}, \phi_{i, R}, \bar{\phi}_{i}, J_{i, L}, J_{i, R}, \bar{J}_{i}\right] \text {, }
$$

and three given values for the source, i.e. $\left[q_{i, L}, q_{i, R}, \bar{q}_{i}\right]$. It is important to notice the transport equation constrains the scalar flux and the current to be continuous at each surface, thus, due to our notation, we add the continuity relations

$$
\begin{aligned}
& \phi_{i, L}=\phi_{i-1, R} \text { and } \phi_{i, R}=\phi_{i+1, L}, \\
& J_{i, L}=J_{i-1, R} \text { and } J_{i, R}=J_{i+1, L} .
\end{aligned}
$$

Note that the source is not constrained to be continuous at the region interfaces. Next, we use the relation

$$
\begin{aligned}
J_{i, R} & =J_{i, R}^{+}-J_{i+1, L}^{+}, \text {and } \\
J_{i, L} & =J_{i-1, R}^{+}-J_{i, L}^{+},
\end{aligned}
$$

to express the acceleration equations (1) and (2) in terms of the partial currents, i.e. $J_{i, R}^{+}=\frac{1}{2} \int_{0}^{1} \mu \psi\left(\frac{\Delta}{2}, \mu\right) d \mu$ and $J_{i, L}^{+}=\frac{1}{2} \int_{-1}^{\sigma}|\mu| \psi\left(-\frac{\Delta}{2}, \mu\right) d \mu$. Thus, Eq. 1 and Eq. (2) become

$$
\begin{aligned}
-J_{i+1, L}^{+}+\left(J_{i, R}^{+}+J_{i, L}^{+}\right)-J_{i-1, R}^{+}+\tau_{i, R} \bar{\phi}_{i} & =\bar{q}_{i} \Delta_{i} \\
\Delta_{i}\left(\frac{J_{i, R}^{+}-J_{i, L}^{+}+J_{i-1, R}^{+}-J_{i+1, L}^{+}}{2}-\bar{J}_{i}\right)+\widetilde{\delta}_{i} \tau_{i, r}\left(\phi_{i, R}-\phi_{i, L}\right) & =\Delta_{i} \widetilde{\delta}_{i}\left(q_{i, R}-q(1, \mathbb{1} 1)\right)
\end{aligned}
$$

\subsection{Closure equations}

After discretizing the acceleration equations 10 and (11), the method has more unknowns than constraints. Thus, we introduce closure relations to constrain our additional unknowns.

First, we assume the surface flux proportional to the sum of the partial surface current, as is commonly the case for pCMR type accelerations, thus

$$
\begin{aligned}
\phi_{i, R} & =\hat{\beta}_{i, R}\left(J_{i, R}^{+}+J_{i+1, L}^{+}\right), \\
\phi_{i, L} & =\hat{\beta}_{i, L}\left(J_{i, L}^{+}+J_{i-1, R}^{+}\right) .
\end{aligned}
$$

Here, the coefficients $\hat{\beta}_{i, L / R}$ are the transport computed parameters and represent the inverse of the albedo coefficients. The underlying assumption for Eq. 
12 is that the distribution of the angular flux within each partial current is fixed at interface. This assumption, in general, is fairly accurate when we have isotropic (or mildly anisotropic) scattering since then the scalar flux will uniformly contributes to all directions of the angular flux, and the contribution of the incoming angular flux distribution to the outgoing angular flux distribution has already been accurately solved for by the transport operator. In the case that we have a $P_{1}$ distribution of the angular flux, $\hat{\beta}_{i, R / L}$ would be equal to 1 and Eq. 12 would be accurate. Finally, using Eq. 14 we assume that $\bar{\phi}_{i}$ is proportional to the sum of the outgoing partial current, as

$$
\bar{\phi}_{i}=\hat{\beta}_{i}\left(J_{i, R}^{+}+J_{i, L}^{+}\right) .
$$

The closure relation (14) uses the same underlying assumption made by Eq. (12) except that we are now applying it over the entire volume of the region. Finally, we link the average current of the region to the boundary spatial current as follows

$$
\bar{J}_{i}=\hat{\alpha}_{i, R} J_{i, R}^{+}-\hat{\alpha}_{i, L} J_{i, L}^{+} .
$$

Eq. 15 assumes a linear relation between $\bar{J}_{i}, J_{i, L}$, and $J_{i, R}$. Note if $\bar{J}_{i} \sim 0$ then $\hat{\alpha}_{i, R}=\hat{\alpha}_{i, L}=1$.

Note that all nonlinear coefficients $\hat{\beta}_{i, R}, \hat{\beta}_{i, L}$, and $\hat{\beta}_{i}$ are set by the inversion of the equations that introduce them, while $\hat{\alpha}_{i, R}$ and $\hat{\alpha}_{i, L}$ are yet to be specified.

\subsubsection{The average current closure relation}

The $\hat{\alpha}_{i, R}$ and $\hat{\alpha}_{i, L}$ coefficients in Eq. (15) are derived by an explicit use of the transport transmission equation. Since the source is constant within the cell, the current depends only from the contribution of incoming angular flux, thus

$$
\bar{J}_{i}=\int_{0}^{1} d \mu \mu\left[\psi_{R}(\mu)-\psi_{L}(\mu)\right]\left(1-e^{-\tau_{i} /|\mu|}\right) .
$$

Relation 16 is modified as

$$
\bar{J}_{i}=\int_{0}^{1} d \mu \mu\left[\psi_{R}(-\mu) \frac{J_{i, R}^{+}}{J_{i, R}^{+}}-\psi_{L}(\mu) \frac{J_{i, L}^{+}}{J_{i, L}^{+}}\right]\left(1-e^{-\tau_{i} /|\mu|}\right),
$$

and by comparing Eq. (17) we obtain the definition for the coefficients $\hat{\alpha}_{i, R}$ and $\hat{\alpha}_{i, L}$, as

$$
\begin{aligned}
\hat{\alpha}_{i, R} & =\frac{\int_{0}^{1} d \mu \mu\left(1-e^{-\tau_{i} /|\mu|}\right) \psi_{R}(-\mu)}{J_{i, R}^{+}}, \text {and } \\
\hat{\alpha}_{i, L} & =\frac{\int_{0}^{1} d \mu \mu\left(1-e^{-\tau_{i} /|\mu|}\right) \psi_{L}(\mu)}{J_{i, L}^{+}},
\end{aligned}
$$

where the integrals are performed with the SN quadrature formula. 


\subsubsection{Consistency with the transport and reduction of the spatial order of the acceleration}

If the transport numerical scheme is conservative, the first equation, i.e. Eq. (1) is satisfied since it is the balance equation enforced by the transport. However, because the parabolic representation of the flux and of the current is an artifact, the second equation is not necessarily satisfied by the transport solution. Therefore we need to use a nonlinear parameter $\hat{\eta}$ to ensure that the second equation is satisfied by the converged solution. Additionally, the current or surface flux terms in the second equation, could go to zero when the flux and currents are flat. Thus, we need to ensure that the relation we choose to represent the parameter $\hat{\eta}$ will not result in an undefined value for these cases. From these requirements, we write Eq. (2) in terms of partial currents

$$
\begin{aligned}
& \Delta_{i}\left(\frac{\left(J_{i, R}^{+}-J_{i, L}^{+}\right)+\left(J_{i-1, R}^{+}-J_{i+1, L}^{+}\right)}{2}-\hat{\alpha}_{i, R} J_{i, R}^{+}+\hat{\alpha}_{i, L} J_{i, L}^{+}\right)+ \\
& \widetilde{\delta}_{i} \tau_{i, r}\left[\hat{\beta}_{i, R}\left(J_{i, R}^{+}+J_{i+1, L}^{+}\right)-\hat{\beta}_{i, L}\left(J_{i, L}^{+}+J_{i-1, R}^{+}\right)\right] \\
= & \Delta_{i} \widetilde{\delta}_{i}\left(q_{i, R}-q_{i, L}\right),
\end{aligned}
$$

and, since the non-linear coefficients together with the partial currents are all positive quantities, we can rearrange Eq. 19 into positive-signed terms $P_{i}$ and negative-signed terms $N_{i}$, obtaining

$$
P_{i}=N_{i}
$$

where

$$
P_{i}=\left(\frac{\Delta_{i}}{2}+\widetilde{\delta}_{i} \tau_{i, r} \hat{\beta}_{i, R}\right) J_{i, R}^{+}+\frac{\Delta_{i}}{2} J_{i-1, R}^{+}+\hat{\alpha}_{i, L} J_{i, L}^{+}+\widetilde{\delta}_{i} \tau_{i, r} \hat{\beta}_{i, R} J_{i+1, L}^{+}+\Delta_{i} \widetilde{\delta}_{i} q_{i, L}
$$

and

$$
N_{i}=\left(\frac{\Delta_{i}}{2}+\widetilde{\delta}_{i} \tau_{i, r} \hat{\beta}_{i, L}\right) J_{i, L}^{+}+\frac{\Delta_{i}}{2} J_{i+1, L}^{+}+\hat{\alpha}_{i, R} J_{i, R}^{+}+\widetilde{\delta}_{i} \tau_{i, r} \hat{\beta}_{i, L} J_{i-1, R}^{+}+\Delta_{i} \widetilde{\delta}_{i} q_{i, R}
$$

We introduce a parameter $\hat{\eta}$ such that the transport satisfies the equation

$$
\hat{\eta}_{i} P_{i}=\left(1-\hat{\eta}_{i}\right) N_{i}
$$

where $\hat{\eta}$ is computed by transport-computed terms (20) and (21) as

$$
\hat{\eta}_{i}=\frac{N_{i}}{P_{i}+N_{i}}
$$


Thus Eq. 19p is rewritten as

$$
\begin{array}{ll} 
& {\left[\hat{\eta}_{i}\left(\frac{\Delta_{i}}{2}+\widetilde{\delta}_{i} \tau_{i, r} \hat{\beta}_{i, R}\right)-\left(1-\hat{\eta}_{i}\right) \hat{\alpha}_{i, R}\right] J_{i, R}^{+}+} \\
& {\left[\hat{\eta}_{i} \frac{\Delta_{i}}{2}-\left(1-\hat{\eta}_{i}\right) \widetilde{\delta}_{i} \tau_{i, r} \hat{\beta}_{i, L}\right] J_{i-1, R}^{+}+} \\
& {\left[\hat{\eta}_{i} \hat{\alpha}_{i, L}-\left(1-\hat{\eta}_{i}\right)\left(\frac{\Delta_{i}}{2}+\widetilde{\delta}_{i} \tau_{i, r} \hat{\beta}_{i, L}\right)\right] J_{i, L}^{+}+} \\
& {\left[\hat{\eta}_{i} \widetilde{\delta}_{i} \tau_{i, r} \hat{\beta}_{i, R}-\left(1-\hat{\eta}_{i}\right) \frac{\Delta_{i}}{2}\right] J_{i+1, L}^{+}} \\
= & \hat{\eta}_{i} \Delta_{i} \widetilde{\delta}_{i} q_{i, R}-\left(1-\hat{\eta}_{i}\right) \Delta_{i} \widetilde{\delta}_{i} q_{i, L}
\end{array}
$$

In the case that the current is almost zero and the flux is slowly varying, the SVRM reduces the spatial order. This is done specifically by modifying the imposed relationship between the current and the scalar flux. The second equation of the SVRM is substituted with the following closure relation,

$$
J_{i, R}^{+}=\hat{r}_{i} J_{i, L}^{+} .
$$

Using Eq. 24 into Eq. (10), and solving Eq. (10) for the flux, it is easy to show that the SVRM method reduces to the CMR method defined by equation 25 .

$$
\hat{g}_{i, R} \bar{\phi}_{i}-\hat{g}_{i+1, L} \bar{\phi}_{i+1}-\hat{g}_{i-1, R} \bar{\phi}_{i-1}+\hat{g}_{i, L} \bar{\phi}_{i}+\left(1-c_{i}\right) \tau_{i} \bar{\phi}_{i}=\bar{q}_{i} \Delta_{i}
$$

where $\hat{g}_{i, R}=\frac{\hat{r}_{i}}{\hat{\beta}_{i}\left(\hat{r}_{i}+1\right)}$ and $\hat{g}_{i, L}=\frac{1}{\hat{\beta}_{i}\left(\hat{r}_{i}+1\right)}$. The new relationship between the current and the scalar flux will be accurate in cases when the outgoing partial current is primarily dependent on the scalar flux. This is often the case in optically thick materials.

\subsection{Boundary conditions}

The last constraints are the boundary conditions. Since the transport operator does not invert the boundary solution with each iteration, until the final solution is reached, there is an erroneous current on the boundary surfaces. Since the final transport solution has to satisfy the boundary conditions, the SVRM method makes use of the "true" boundary conditions instead of using a nonlinear albedo coefficient as is commonly done in the CMR and CMFD. This is a way of achieving additional acceleration on the boundary. The boundary conditions can be expressed in a general form by Eqs. 26) and (27) for the left and right sides respectively,

$$
\begin{array}{ll}
J_{0, R}^{+,(n+1)}=v_{L \leftarrow L} J_{1, L}^{+,(n+1)}+v_{L \leftarrow R} J_{I, R}^{+,(n+1)} & \text { for } i=1, \\
J_{I+1, L}^{+,(n+1)}=v_{R \leftarrow L} J_{1, L}^{+,(n+1)}+v_{R \leftarrow R} J_{I, R}^{+,(n+1)} & \text { for } i=I .
\end{array}
$$


Here, the currents $J_{0, R}^{+,(n+1)}$ and $J_{I+1, L}^{+,(n+1)}$ indicate the incoming boundary current on the left and on the right sides respectively. The albedo coefficients $v_{L \leftarrow L}$, $v_{L \leftarrow R}, v_{R \leftarrow L}$, and $v_{R \leftarrow R}$ are defined by Table 28.

\begin{tabular}{|c|c|c|c|c|}
\hline & Reflective & Periodic & Vacuum & Fourier periodic \\
\hline$v_{L \leftarrow L}=$ & 1 & 0 & 0 & 0 \\
\hline$v_{L \leftarrow R}=$ & 0 & 1 & 0 & $e^{-j \frac{2 \pi \zeta}{\Delta_{1}}\left(x_{1}-\frac{\Delta_{1}}{2}\right)}$ \\
\hline$v_{R \leftarrow L}=$ & 0 & 1 & 0 & $e^{-j \frac{2 \pi \zeta}{\Delta_{I}}\left(x_{I}+\frac{\Delta_{I}}{2}\right)}$ \\
\hline$v_{R \leftarrow R}=$ & 1 & 0 & 0 & 0 \\
\hline
\end{tabular}

\subsection{Matrix form of SVRM}

The SVRM equations 10 and 111 can be cast in a matrix form of the type

$$
(\hat{A}+\hat{B}) J=Q_{A c}
$$

where $\hat{A}$ counts for internal contribution from regions while $\hat{B}$ counts for boundary condition and $Q_{A c}$ for the source. More specifically, for a node $i$ the equations take a $2 \times 2$ matrix form of the type

$$
\hat{A}_{i, i-1} J_{i-1}+\hat{A}_{i, i} J_{i}+\hat{A}_{i, i+1} J_{i+1}=Q_{A c, i} \quad \text { when } i \neq 1 \text { and } i \neq I
$$

where

$$
J_{i}=\left[\begin{array}{c}
J_{i, L}^{+} \\
J_{i, R}^{+}
\end{array}\right] \text {and } Q_{A c, i}=\left[\begin{array}{c}
\Delta_{i} \bar{q}_{i} \\
\Delta_{i} \widetilde{\delta}_{i}\left(\widehat{\eta} q_{i, R}-(1-\widehat{\eta}) q_{i, L}\right)
\end{array}\right]
$$

By substituting in the closure relations, Eqs. (15)-(14), into Eq. 6 and using Eq. 23, we get an expression for the $2 \times 2$ matrices $A_{i, i-1}, \hat{A}_{i, i}$ and $\hat{A}_{i, i+1}$ for $i=1, \ldots, I$,

$$
\begin{aligned}
& \hat{A}_{i, i-1}=\left[\begin{array}{cc}
0 & -1 \\
0 & \hat{\eta}_{i} \frac{\Delta_{i}}{2}-\left(1-\hat{\eta}_{i}\right) \widetilde{\delta}_{i} \tau_{i, r} \hat{\beta}_{i, L}
\end{array}\right] \\
& \hat{A}_{i, i}=\left[\begin{array}{cc}
1+\hat{\beta}_{i} \tau_{i, R} & 1+\hat{\beta}_{i} \tau_{i, R} \\
{\left[\hat{\eta}_{i} \hat{\alpha}_{i, L}-\left(1-\hat{\eta}_{i}\right)\left(\frac{\Delta_{i}}{2}+\widetilde{\delta}_{i} \tau_{i, r} \hat{\beta}_{i, L}\right)\right]} & {\left[\hat{\eta}_{i}\left(\frac{\Delta_{i}}{2}+\widetilde{\delta}_{i} \tau_{i, r} \hat{\beta}_{i, R}\right)-\left(1-\hat{\eta}_{i}\right) \hat{\alpha}_{i, R}\right]}
\end{array}\right], \\
& \hat{A}_{i, i+1}=\left[\begin{array}{cc}
-1 & 0 \\
\hat{\eta}_{i} \widetilde{\delta}_{i} \tau_{i, r} \hat{\beta}_{i, R}-\left(1-\hat{\eta}_{i}\right) \frac{\Delta_{i}}{2} & 0
\end{array}\right]
\end{aligned}
$$

and finally for $\hat{A}$,

$$
\hat{A}=\left[\begin{array}{ccccc}
\hat{A}_{1,1} & \hat{A}_{1,2} & 0 & \ldots & 0 \\
\ddots & \ddots & \ddots & \ddots & \vdots \\
0 & \hat{A}_{i, i-1} & \hat{A}_{i, i} & \hat{A}_{i, i+1} & 0 \\
\vdots & \ddots & \ddots & \ddots & \ddots \\
0 & \ldots & 0 & \hat{A}_{I, I-1} & \hat{A}_{I, I}
\end{array}\right]
$$


The matrix $\widehat{B}$ of the boundary conditions is obtained by applying Eq. 26. and (26) to Eq. (30) written for the cells $i=1$ and $i=I$, that holds

$$
\hat{B}=\left[\begin{array}{ccccc}
\hat{A}_{1,0} \hat{B}_{L, 1} & 0 & \ldots & 0 & \hat{A}_{1,0} \hat{B}_{L, I} \\
0 & 0 & \ldots & 0 & 0 \\
\vdots & \vdots & \ddots & \vdots & \vdots \\
0 & 0 & \ldots & 0 & 0 \\
\hat{A}_{I, I+1} \hat{B}_{R, 1} & 0 & \ldots & 0 & \hat{A}_{I, I+1} \hat{B}_{R, I}
\end{array}\right]
$$

where

$$
\begin{aligned}
\hat{B}_{L, 1} & =\left[\begin{array}{cc}
0 & 0 \\
v_{L \leftarrow L} & 0
\end{array}\right], \\
\hat{B}_{L, I} & =\left[\begin{array}{cc}
0 & 0 \\
0 & v_{L \leftarrow R}
\end{array}\right], \\
\hat{B}_{R, 1} & =\left[\begin{array}{cc}
v_{R \leftarrow L} & 0 \\
0 & 0
\end{array}\right], \\
\hat{B}_{R, I} & =\left[\begin{array}{cc}
0 & v_{R \leftarrow R} \\
0 & 0
\end{array}\right] .
\end{aligned}
$$

\section{Defining the Transport Operator}

For the sake of the simplicity we will consider an $S_{2}$-like formalism considering the index for the angular direction as 1 for directions with $\mu>0$ and 2 for directions with $\mu<0$. Because of the linearity of the transport operator, the interior scalar flux and the outgoing surface fluxes can be expressed like a linear combination of the sources, from scattering and external fixed sources, and the incoming fluxes. In the case of

$$
\begin{aligned}
\psi_{i, R, 1} & =a_{i} \psi_{i-1, R, 1}+\frac{b_{i}}{2} \phi_{i}+\frac{b_{i}^{\prime}}{2} q_{i}, \\
\psi_{i, L, 2} & =a_{i} \psi_{i+1, L, 2}+\frac{b_{i}}{2} \phi_{i}+\frac{b_{i}^{\prime}}{2} q_{i}, \\
\bar{\phi}_{i} & =k_{i}\left(\psi_{i, L, 1}+\psi_{i, R, 2}\right)+d_{i} \phi_{i}+d_{i}^{\prime} q_{i}
\end{aligned}
$$

- $\psi_{i, L, 1 / 2}$ is the incoming flux over the left side of the mesh and for directions of the first/second quadrant,

- $\psi_{i, R, 1 / 2}$ is the incoming flux over the left side of the mesh and for directions of the first/second quadrant,

- $\bar{\phi}_{i}$ is the average scalar flux,

- $q_{i}$ is the average fixed source in the region $i$, and 
- $\left(a_{i}, b_{i}, b_{i}^{\prime}, k_{i}, d_{i}, d_{i}^{\prime}\right)$ are the numerical transport coefficients of the region $i$ that depends on $|\mu|$. In particular, $a_{i}, b_{i}$ and $k_{i}$ are coefficients if we use an $S_{2}$ quadrature formula, while they diagonal matrices of dimension $\frac{N}{2}$ for an arbitrary $S_{N}$ with $N>2$. For the sake of simplicity we will use an $S_{2}$ formalism. For the notation being used we have to enforce the continuity relation for the incoming angular flux, i.e. $\psi_{i, L, 1}=\psi_{i-1, R, 1}$ and $\psi_{i, R, 2}=\psi_{i+1, L, 2}$.

If we consider the $\Psi_{i}$ as the vector containing the unknown for region $i$, i.e. the scalar flux plus the outgoing angular fluxes, we get

$$
\Psi_{i}=\left[\begin{array}{c}
\psi_{i, L, 2} \\
\psi_{i, R, 1} \\
\underline{\phi}_{i}
\end{array}\right]
$$

and we can express Eqs. 411-43 as

$$
\Psi_{i}=T_{i, i-1} \Psi_{i-1}+T_{i, i} \Psi_{i}+T_{i, i+1} \Psi_{i+1}+Q_{i}^{\prime}
$$

where

$$
\begin{aligned}
T_{i, i} & =\left[\begin{array}{ccc}
0 & 0 & b_{i} / 2 \\
0 & 0 & b_{i} / 2 \\
0 & 0 & d_{i}
\end{array}\right], \\
T_{i, i-1} & =\left[\begin{array}{ccc}
0 & 0 & 0 \\
0 & a_{i} & 0 \\
0 & k_{i} & 0
\end{array}\right], \\
T_{i, i+1} & =\left[\begin{array}{ccc}
a_{i} & 0 & 0 \\
0 & 0 & 0 \\
k_{i} & 0 & 0
\end{array}\right]
\end{aligned}
$$

are the transport matrices for the region $i$, and $Q_{i}^{\prime}$

$$
Q_{i}^{\prime}=\left[\begin{array}{l}
b_{i}^{\prime} \frac{q}{2} \\
b_{i}^{\prime} \frac{q}{2} \\
d_{i}^{\prime} q
\end{array}\right]
$$

is the contribution from the fixed source. We can distinguish two iterative schemes, i.e. the Gauss-Jacobi (G-J) and Gauss-Seidel (G-S) respectively, by simply specialize Eq. 44 as

$$
\Psi_{i}^{(n+1 / 2)}=T_{i, i-1} \Psi_{i-1}^{(n)}+T_{i, i} \Psi_{i}^{(n)}+T_{i, i+1} \Psi_{i+1}^{(n)}+Q_{i}^{\prime},
$$

for the pure G-J scheme, and

$$
-T_{i, i-1} \Psi_{i-1}^{(n+1 / 2)}+\Psi_{i}^{(n+1 / 2)}-T_{i, i+1} \Psi_{i+1}^{(n+1 / 2)}=T_{i, i} \Psi_{i}^{(n)}+Q_{i}^{\prime},
$$

for the G-S scheme. More, we can define the global transport matrix for the G-J scheme as

$$
\left.T\right|_{G J}=T_{l}+T_{d}+T_{u}
$$


where the $T_{d}$ is the block-diagonal matrix composed by the matrices $T_{i, i}$, while $T_{l}$ and $T_{u}$ are respectively the lower and the upper triangular matrix composed by the block $T_{i, i-1}$ and $T_{i, i+1}$, respectively. Thus the transport matrix for the $\mathrm{G}-\mathrm{S}$ is

$$
\left.T\right|_{G S}=\left(I-T_{l}-T_{u}\right)^{-1} T_{d}
$$

As a consequence, the source vector for the $G S$ will be $\left.Q^{\prime}\right|_{G S}=\left(I-T_{l}\right.$ $\left.T_{u}\right)^{-1} Q^{\prime}$. As mentioned in the introduction, the spatial transport discretization is the method of characteristics (MOC) with step approximation of the source. By defining $c_{i}=\frac{\Sigma_{s, i}}{\Sigma_{t, i}}$ as the scattering ratio and $\tau_{i}=\Sigma_{t, i} \Delta_{i}$ as the optical thickness, the transport coefficients $\left(a_{i}, b_{i}, b_{i}^{\prime}, k_{i}, d_{i}, d_{i}^{\prime}\right)$ for the MOC are

$$
\begin{aligned}
a_{i} & =e^{-\frac{\tau_{i}}{|\mu|}}, \\
b_{i} & =c_{i}\left(1-a_{i}\right), \\
k_{i} & =\frac{|\mu|\left(1-a_{i}\right)}{\tau_{i}}, \\
d_{i} & =c_{i}\left(1-k_{i}\right), \\
b_{i}^{\prime} & =\frac{b_{i}}{\Sigma_{s, i}}, \\
d_{i}^{\prime} & =\frac{d_{i}}{\Sigma_{s, i}} .
\end{aligned}
$$

\subsection{Boundary conditions}

As for the acceleration equations, we introduce two fictitious regions, the region 0 and region $I+1$ to solve for the boundary fluxes. Those two regions updates the incoming angular flux on the left an on the right boundary. As before, we can generalize the boundary conditions by defining the two equations, the first on the left boundary

$$
\psi_{0, R, 1}^{(n+1)}=v_{L \leftarrow L} \psi_{1, L, 2}^{(n)}+v_{L \leftarrow R} \psi_{I, R, 1}^{(n)} \quad \text { for } i=1,
$$

and the second on the right boundary

$$
\psi_{I+1, L, 2}^{(n+1)}=v_{R \leftarrow L} \psi_{1, L, 2}^{(n)}+v_{R \leftarrow R} \psi_{I, R, 1}^{(n)} \quad \text { for } i=I .
$$

In particular, the albedo coefficients $v_{L \leftarrow L}, v_{L \leftarrow R}, v_{R \leftarrow L}$, and $v_{R \leftarrow R}$ assumes the values contained in (53).

\begin{tabular}{|c|c|c|c|c|}
\hline & Reflective & Periodic & Vacuum & Fourier periodic \\
\hline$v_{L \leftarrow L}=$ & 1 & 0 & 0 & 0 \\
\hline$v_{L \leftarrow R}=$ & 0 & 1 & 0 & $e^{-j \frac{2 \pi \zeta}{\Delta_{1}}\left(x_{1}-\frac{\Delta_{1}}{2}\right)}$ \\
\hline$v_{R \leftarrow L}=$ & 0 & 1 & 0 & $e^{-j \frac{2 \pi \zeta}{\Delta_{I}}\left(x_{I}+\frac{\Delta_{I}}{2}\right)}$ \\
\hline$v_{R \leftarrow R}=$ & 1 & 0 & 0 & 0 \\
\hline
\end{tabular}


Using this formalism, if we specialize Eq. 477 for the first region by taking into account the left-side boundary condition, i.e. Eq. (51), we obtain

$$
\Psi_{1}^{(n+1 / 2)}=T_{1,0} V_{L \leftarrow R} \Psi_{I}^{(n)}+\left(T_{1,0} V_{L \leftarrow L}+T_{1,1}\right) \Psi_{1}^{(n)}+T_{1,2} \Psi_{2}^{(n)}+Q_{i}^{\prime},
$$

where

$$
\begin{aligned}
V_{L \leftarrow L} & =\left[\begin{array}{ccc}
0 & 0 & 0 \\
v_{L \leftarrow L} & 0 & 0 \\
0 & 0 & 0
\end{array}\right], \\
V_{L \leftarrow R}= & {\left[\begin{array}{ccc}
0 & 0 & 0 \\
0 & v_{L \leftarrow R} & 0 \\
0 & 0 & 0
\end{array}\right] . }
\end{aligned}
$$

We do the same for the right boundary condition and we obtain

$$
\Psi_{I}^{(n+1 / 2)}=T_{I, I+1} V_{R \leftarrow L} \Psi_{1}^{(n)}+\left(T_{I, I+1} V_{R \leftarrow R}+T_{1,1}\right) \Psi_{1}^{(n)}+T_{1,2} \Psi_{2}^{(n)}+Q_{i}^{\prime},
$$

where

$$
\begin{aligned}
V_{R \leftarrow R}= & {\left[\begin{array}{ccc}
0 & v_{R \leftarrow R} & 0 \\
0 & 0 & 0 \\
0 & 0 & 0
\end{array}\right], } \\
V_{R \leftarrow L}= & {\left[\begin{array}{ccc}
v_{R \leftarrow L} & 0 & 0 \\
0 & 0 & 0 \\
0 & 0 & 0
\end{array}\right] . }
\end{aligned}
$$

\subsection{The discrete-to-moment operator and the prolonga- tion operator}

The acceleration space needs the transport angular moments. The mapping from the transport angular flux and transport angular moments is given by the so-called discrete-to-moment operator. In the specific case, the discreteto-moment operator maps both volume and interface angular moments. In coherence with our $\mathrm{S}_{2}$-like notation, the angular moments that defines the acceleration space are the outgoing partial interface current and the outgoing partial interface flux, namely $J_{i, L / R}^{+}$and $\phi_{i, L / R}^{+}$, and the average volume current and flux, respectively $\bar{J}_{i}$ and $\bar{\phi}_{i}$,

$$
\begin{aligned}
J_{i, L}^{+} & =w|\mu| \psi_{i, L, 2} \\
J_{i, R}^{+} & =w|\mu| \psi_{i, R, 1} \\
\bar{J}_{i} & =w|\mu| k_{i}\left(\psi_{i-1, R, 1}-\psi_{i+1, L, 2}\right) \\
\phi_{i, L} & =w\left(\psi_{i-1, R, 1}+\psi_{i, L, 2}\right) \\
\phi_{i, R} & =w\left(\psi_{i, R, 1}+\psi_{i+1, L, 2}\right) \\
\bar{\phi}_{i} & =\bar{\phi}_{i}
\end{aligned}
$$


by making the positions

$$
\Psi_{i}=\left[\begin{array}{c}
\psi_{i, L, 2} \\
\psi_{i, R, 1} \\
\phi_{i}
\end{array}\right], \text { with } \Psi=\left\{\Psi_{i}\right\}_{i=1, I},
$$

and

$$
\Phi_{i}=\left[\begin{array}{c}
J_{i, L}^{+} \\
J_{i, R}^{+} \\
J_{i} \\
\phi_{i, L}^{+} \\
\phi_{i, R}^{+} \\
\phi_{i}
\end{array}\right], \text { with } \Phi=\left\{\Phi_{i}\right\}_{i=1, I}
$$

we can write the discrete-to-moment operator as

$$
\Phi=K \Psi
$$

where $K$ is the block-rectangular matrix

$$
K=\left[\begin{array}{ccccc}
K_{1,1} & K_{1,2} & 0 & \ldots & 0 \\
0 & \ddots & \ddots & \ddots & \vdots \\
0 & K_{i, i-1} & K_{i, i} & K_{i, i+1} & 0 \\
\vdots & \ddots & \ddots & \ddots & 0 \\
0 & \ldots & 0 & K_{I-1, I} & K_{I, I}
\end{array}\right]
$$

where the minors $K_{i, i-1}, K_{i, i}$ and $K_{i, i+1}$ satisfy the equation

$$
\Phi_{i}=K_{i, i-1} \Psi_{i-1}+K_{i, i} \Psi_{i}+K_{i, i+1} \Psi_{i+1},
$$

such that

$$
\begin{aligned}
K_{i, i}= & {\left[\begin{array}{ccc}
w|\mu| & 0 & 0 \\
0 & w|\mu| & 0 \\
0 & 0 & 0 \\
w & 0 & 0 \\
0 & w & 0 \\
0 & 0 & 1
\end{array}\right], } \\
K_{i, i-1}= & {\left[\begin{array}{ccc}
0 & 0 & 0 \\
0 & 0 & 0 \\
0 & w k_{i}|\mu| & 0 \\
0 & w & 0 \\
0 & 0 & 0 \\
0 & 0 & 0
\end{array}\right], \text { and } } \\
K_{i, i+1}= & {\left[\begin{array}{ccc}
0 & 0 & 0 \\
0 & 0 & 0 \\
0 & w k_{i}|\mu| & 0 \\
0 & 0 & 0 \\
w & 0 & 0 \\
0 & 0 & 0
\end{array}\right] . }
\end{aligned}
$$


In order to accurately update the transport solution using the SVRM partial current, we must preserve the assumptions of the closure equations. Since the angular flux distribution within each partial current was fixed by the closure relation (12), the updated angular flux must be proportional to the partial current. In the prolongation operator, the couple of values $\left(J_{i, L}^{+}, J_{i, R}^{+}\right)$is used to correct both interface angular flux and scalar flux. As is common for a rebalancing method, the SVRM reconstructs the scalar flux using Eq. 14 with the SVRM-computed partial currents,

$$
\bar{\phi}_{i}^{(n+1)}=\hat{\beta}_{i}\left(J_{i, R}^{+}+J_{i, L}^{+}\right)^{(n+1)} .
$$

The second update is on the interface angular flux,

$$
\begin{aligned}
\psi_{i, L, 2}^{(n+1)} & =\frac{J_{i, L}^{+,(n+1)}}{J_{i, L}^{+,(n+1 / 2)}} \psi_{1, L, 2}^{(n+1 / 2)}, \\
\psi_{i, R, 1}^{(n+1)} & =\frac{J_{i, R}^{+,(n+1)}}{J_{i, R}^{+,(n+1 / 2)}} \psi_{1, R, 2}^{(n+1 / 2)} .
\end{aligned}
$$

Eqs. (62) and (63) are useful when the transport sweep is done using a G-J iterative scheme, which commonly used to access domain decomposition and, thus, parallel computing. Eqs. 62 and (63) can be cast in a matrix formalism,

$$
\Psi^{(n+1)}=\widehat{P} J^{(n+1)},
$$

where $\widehat{P}$ is a rectangular diagonal-by-block matrix composed of matrix $\widehat{P}_{i}$ containing the coefficients of Eqs. 61), 62 and (63) for each region $i$,

$$
\widehat{P}_{i}=\left[\begin{array}{cc}
\frac{\psi_{i, L, 2}^{(n+1 / 2)}}{J_{i, L}^{+,(n+1 / 2)}} & 0 \\
0 & \frac{\psi_{i, R, 1}^{(n+1 / 2)}}{J_{i, R}^{+,(n+1 / 2)}} \hat{\beta}_{i}
\end{array}\right] .
$$

We remark that in this paper the SVRM mesh is the same as for the transport operator. This choice simplifies the spectral analysis of the method. Nevertheless, the implementation of the SVRM is meant to treat coarser spatial mesh.

\section{The Jacobian of the iterative scheme}

Since we have an iterative multivariable map of the form $\Psi^{(n+1)}=F\left(\Psi^{(n)}\right)$, we can express the Jacobian in a point $X$ as

$$
J=\left.\nabla_{\Psi^{(n)}} F\left(\Psi^{(n)}\right)\right|_{\Psi^{(n)}=X} .
$$


The nonlinear scheme $F$ summarizes the following system of equations

$$
\left\{\begin{array}{c}
\Psi^{(n+1 / 2)}=T \Psi^{(n)}+Q^{\prime}, \\
\Phi^{(n+1 / 2)}=K \Psi^{(n+1 / 2)}, \\
\widehat{A}\left(\Phi^{(n+1 / 2)}\right) J^{(n+1)}=Q_{A}, \\
\Psi^{(n+1)}=\widehat{P}\left(\Psi^{(n+1 / 2)}, \Phi^{(n+1 / 2)}\right) J^{(n+1)} .
\end{array}\right.
$$

where we explicitly write the non-linear nature of the matrix $\widehat{A}\left(\Phi^{(n+1 / 2)}\right)$ and $\widehat{\widehat{P}}\left(\Psi^{(n+1 / 2)}, \Phi^{(n+1 / 2)}\right)$. The first equation of 66 is the transport source iteration, where $T$ is the transport matrix given by 48 or 49 , while $\Psi^{(n)}$ is the vector containing the transport iterative unknowns. The second equations is a the of discrete-to-moment linear operator mapping the transport unknowns to the angular moments. Here, $K$ is the rectangular matrix defined in map (58) and $\Phi^{(n+1 / 2)}$ is the vectors of transport angular moments. The third equation is the acceleration equation with $\widehat{A}\left(\Phi^{(n+1 / 2)}\right)$ as the non-linear operator, $J^{(n+1)}$ as the unknown partial currents and $Q_{A}$ as the acceleration source. Next, the forth equation is the correction equation that updates the transport unknowns $\Psi^{(n+1)}$. The equation is defined by the non-linear correction matrix $\widehat{P}\left(\Phi^{(n+1 / 2)}\right)$ defined in (64) and (65).

The investigation of the rate of convergence of the system (66) can be carried out by studying the Jacobian at the fixed point $\Psi^{(\infty)}=\Lambda$, as

$$
E=\left.\nabla_{\Psi^{(n)}} \Psi^{(n+1)}\right|_{\Psi^{(n)}=\Lambda} .
$$

Since

$$
\nabla_{\Psi^{(n)}} \Psi^{(n+1)}=\nabla_{\Phi^{(n+1 / 2)}} \Psi^{(n+1)} \nabla_{\Psi^{(n)}} \Phi^{(n+1 / 2)},
$$

the calculation of the Jacobian can be expressed as the composition of the linear transport matrices

$$
\nabla_{\Psi^{(n)}} \Phi^{(n+1 / 2)}=K T
$$

and the non-linear matrices

$$
\begin{aligned}
\nabla_{\Phi^{(n+1 / 2)}} \Psi^{(n+1)}= & \nabla_{\Phi^{(n+1 / 2)}}\left[\widehat{P}\left(\Phi^{(n+1 / 2)}\right) \widehat{A}^{-1} Q_{A}\right]+ \\
& \widehat{P} \nabla_{\Phi^{(n+1 / 2)}}\left[\widehat{A}^{-1}\left(\Phi^{(n+1 / 2)}\right) Q_{A}\right],
\end{aligned}
$$

where we explicitly shows the non-linear dependence of the matrices $\widehat{P}$ and $\widehat{A}^{-1}$ when the gradient $\nabla_{\Phi^{(n+1 / 2)}}$ is applied. Combining Eq. 69 and 70 into Eq. (68), we obtain the expression for the Jacobian in function of matrices $K, T, \widehat{A}^{-1}, \widehat{P}$ and of the source $Q_{A}$. It is also clear that the effectiveness of the non-linear equation is governed by the largest eigenvalue of the matrix $\nabla_{\Phi^{(n+1 / 2)}} \Psi^{(n+1)}$. In fact, neglecting the boundary conditions, we have the 
bounding relation $\|K T\|<c=\max \left\{\frac{\Sigma_{s}}{\Sigma}\right\}<1$ and, thus, the Jacobian norm will be bounded by

$$
\|E\|<\lambda c
$$

with $\lambda$ being the largest eigenvalue of the matrix $\nabla_{\Phi^{(n+1 / 2)}} \Psi^{(n+1)}$.

We focus our analysis on two iterative transport schemes. The first is the classical transport sweep, where the spatial meshes are swept one after the other following the direction of flight of the particles. In this case, the iterative scheme is a block Gauss-Jacobi over the angular directions, where each block represent the spatial unknowns, and a Gauss-Seidel over the space. In that case, the vector $\Psi$ contains the scalar fluxes $\left\{\phi_{i}\right\}_{i=1, I}$ and, in the case of homogeneous boundary conditions, the outgoing angular fluxes $\psi_{1, L, 2}$ and $\psi_{I, R, 1}$ needed for updating boundary conditions, i.e. $\Psi=\left\{\left\{\phi_{i}\right\}_{i=1, I}, \psi_{1, L, 2}, \psi_{I, R, 1}\right\}$. The second is a pure Gauss-Jacobi scheme where the vector of the unknowns, $\Psi$, contains the scalar fluxes and the outgoing angular fluxes of each computational mesh, i.e. $\Psi=\left\{\phi_{i}, \psi_{i, L, 2}, \psi_{i, R, 1}\right\}_{i=1, I}$. This second scheme is typically used in the parallelism framework for accessing scalability, as is typically done in the domain decomposition method.

In the following we will study the convergence properties of the two transport iterative schemes, and the capability of the acceleration to enhance the convergence speed.

\subsection{Computing the Jacobian}

The starting point is Eq. (68), the Jacobian is a composition of partial derivatives and this Section is dedicated to the tedious computation of the derivatives. Since $\frac{\partial \Phi^{(n+1 / 2)}}{\partial \Psi^{(n)}}=K T$ is the transport contribution to the Jacobian, the acceleration contribution, which is the matrix (70), can be rewritten as

$$
\frac{\partial \Psi^{(n+1)}}{\partial \Phi^{(n+1 / 2)}}=\widehat{P} \frac{\partial J^{(n+1)}}{\partial \Phi^{(n+1 / 2)}}+\frac{\partial\left(\widehat{P} J^{(n+1)}\right)}{\partial \Phi^{(n+1 / 2)}} .
$$

The matrix $\frac{\partial J^{(n+1)}}{\partial \Phi^{(n+1 / 2)}}$ is obtained by deriving of the SVRM equations, i.e. the third equation in the system (66), obtaining

$$
\frac{\partial J^{(n+1)}}{\partial \Phi^{(n+1 / 2)}}=\widehat{A}^{-1} \frac{\partial\left[\widehat{A} J^{(n+1)}\right]}{\partial \Phi^{(n+1 / 2)}} .
$$

We can explicitly compute the $\frac{\partial\left[\widehat{A} J^{(n+1)}\right]}{\partial \Phi^{(n+1 / 2)}}$ matrix as a composition of partial derivatives

$$
\frac{\partial\left[\widehat{A} J^{(n+1)}\right]}{\partial \Phi^{(n+1 / 2)}}=\sum_{x \in\left(\hat{\alpha}_{i, R}, \hat{\alpha}_{i, L}, \hat{\beta}_{i, R}, \hat{\beta}_{i, L}, \hat{\beta}_{i}, \widehat{\eta}_{i}, \widehat{\gamma}_{R}, \widehat{\gamma}_{L}\right)_{i=1, I}} \frac{\partial\left[\widehat{A} J^{(n+1)}\right]}{\partial x} \otimes \frac{\partial x}{\partial \Phi^{(n+1 / 2)}},
$$


note that in Eq. $73 J^{(n+1)}$ is not derived. The non-zero derivatives that appears in 73 are

$$
\begin{aligned}
& \frac{\partial \hat{A}_{i, i}}{\partial \hat{\alpha}_{i, L}}=\left[\begin{array}{cc}
0 & 0 \\
\hat{\eta}_{i} & 0
\end{array}\right], \\
& \frac{\partial \hat{A}_{i, i}}{\partial \hat{\alpha}_{i, R}}=\left[\begin{array}{cc}
0 & 0 \\
0 & -\left(1-\hat{\eta}_{i}\right)
\end{array}\right] \text {, } \\
& \frac{\partial \hat{A}_{i, i+1}}{\partial \hat{\beta}_{i, R}}=\left[\begin{array}{cc}
0 & 0 \\
\hat{\eta}_{i} \widetilde{\delta}_{i} \tau_{i, r} & 0
\end{array}\right], \\
& \frac{\partial \hat{A}_{i, i}}{\partial \hat{\beta}_{i, R}}=\left[\begin{array}{cc}
0 & 0 \\
0 & \hat{\eta}_{i} \widetilde{\delta}_{i} \tau_{i, r}
\end{array}\right] \text {, } \\
& \frac{\partial \hat{A}_{i, i-1}}{\partial \hat{\beta}_{i, L}}=\left[\begin{array}{cc}
0 & 0 \\
0 & -\left(1-\hat{\eta}_{i}\right) \widetilde{\delta}_{i} \tau_{i, r}
\end{array}\right] \\
& \frac{\partial \hat{A}_{i, i}}{\partial \hat{\beta}_{i, L}}=\left[\begin{array}{cc}
0 & 0 \\
-\left(1-\hat{\eta}_{i}\right) \widetilde{\delta}_{i} \tau_{i, r} & 0
\end{array}\right], \\
& \frac{\partial \hat{A}_{i, i}}{\partial \hat{\beta}_{i}}=\left[\begin{array}{cc}
\tau_{i, R} & \tau_{i, R} \\
0 & 0
\end{array}\right] \\
& \frac{\partial \hat{A}_{i, i}}{\partial \hat{\eta}_{i}}=\left[\begin{array}{cc}
0 & 0 \\
\left(\hat{\alpha}_{i, L}+\frac{\Delta_{i}}{2}+\widetilde{\delta}_{i} \tau_{i, r} \hat{\beta}_{i, L}\right) & \left(\hat{\alpha}_{i, R}+\frac{\Delta_{i}}{2}+\widetilde{\delta}_{i} \tau_{i, r} \hat{\beta}_{i, R}\right)
\end{array}\right], \\
& \frac{\partial \hat{A}_{i, i-1}}{\partial \hat{\eta}_{i}}=\left[\begin{array}{cc}
0 & 0 \\
0 & \frac{\Delta_{i}}{2}+\widetilde{\delta}_{i} \tau_{i, r} \hat{\beta}_{i, L}
\end{array}\right] \text { and } \\
& \frac{\partial \hat{A}_{i, i+1}}{\partial \hat{\eta}_{i}}=\left[\begin{array}{cc}
0 & 0 \\
\frac{\Delta_{i}}{2}+\widetilde{\delta}_{i} \tau_{i, r} \hat{\beta}_{i, R} & 0
\end{array}\right] \text {, }
\end{aligned}
$$

In Eq. 73 , the derivatives of the non-linear coefficients $\left(\hat{\alpha}_{i, R}, \hat{\alpha}_{i, L}, \hat{\beta}_{i, R}, \hat{\beta}_{i, L}, \hat{\beta}_{i}, \widehat{\eta}_{i}, \widehat{\gamma}_{R}, \widehat{\gamma}_{L}\right)_{i=1, I}$ with respect to the angular flux moments $\Phi_{i}^{(n+1 / 2)}$ are

$$
\begin{gathered}
\frac{\partial \hat{\alpha}_{i, R}}{\partial \Phi_{i}^{(n+1 / 2)}}=\frac{1}{J_{i, R}^{(n+1 / 2)}}\left[0,-\hat{\alpha}_{i, R}, 0,0,0,0\right], \\
\frac{\partial \hat{\alpha}_{i, L}}{\partial \Phi_{i}^{(n+1 / 2)}}=\frac{1}{J_{i, L}^{(n+1 / 2)}}\left[-\hat{\alpha}_{i, L}, 0,0,0,0,0\right], \\
\frac{\partial \hat{\beta}_{i, R}}{\partial \Phi_{i}^{(n+1 / 2)}}=\frac{1}{\left(J_{i, R}^{+}+J_{i+1, L}^{+}\right)^{(n+1 / 2)}}\left[\begin{array}{lllll}
0 & -\hat{\beta}_{i, R}, 0,0, & 0 & 0
\end{array}\right], \\
\frac{\partial \hat{\beta}_{i, R}}{\partial \Phi_{i+1}^{(n+1 / 2)}}=\frac{1}{\left(J_{i, R}^{+}+J_{i+1, L}^{+,}\right)^{(n+1 / 2)}}\left[-\hat{\beta}_{i, R}, 0,0,0,0,0\right],
\end{gathered}
$$




$$
\begin{aligned}
\frac{\partial \hat{\beta}_{i, L}}{\partial \Phi_{i}^{(n+1 / 2)}} & =\frac{1}{\left(J_{i, L}^{+}+J_{i-1, R}^{+,}\right)^{(n+1 / 2)}}\left[-\hat{\beta}_{i, L}, 0,0,1,0,0\right] \\
\frac{\partial \hat{\beta}_{i, L}}{\partial \Phi_{i-1}^{(n+1 / 2)}} & =\frac{1}{\left(J_{i, L}^{+}+J_{i-1, R}^{+,}\right)^{(n+1 / 2)}}\left[0,-\hat{\beta}_{i, L}, 0,0,0,0\right] \\
\frac{\partial \hat{\beta}_{i}}{\partial \Phi_{i}^{(n+1 / 2)}} & =\frac{1}{\left(J_{R}^{+}+J_{L}^{+}\right)^{(n+1 / 2)}}\left[-\hat{\beta}_{i},-\hat{\beta}_{i}, 0,0,0,0\right], \\
\frac{\partial \widehat{\eta}_{i}}{\partial \Phi^{(n+1 / 2)}}= & -\frac{1}{\left(P_{i}+N_{i}\right)}\left[\left(1-\widehat{\eta}_{i}\right) \frac{\partial P_{i}}{\partial \Phi^{(n+1 / 2)}}+\widehat{\eta}_{i} \frac{\partial N_{i}}{\partial \Phi^{(n+1 / 2)}}\right]
\end{aligned}
$$

where the partial derivatives for $P_{i}$ and $N_{i}$ are

$$
\begin{aligned}
& \frac{\partial P_{i}}{\partial \Phi_{i}^{(n+1 / 2)}}=\left[\hat{\alpha}_{i, L},\left(\frac{\Delta_{i}}{2}+\widetilde{\delta}_{i} \tau_{i, r} \hat{\beta}_{i, R}\right), 0,0,0,0\right], \\
& \frac{\partial P_{i}}{\partial \Phi_{i-1}^{(n+1 / 2)}}=\left[0, \frac{\Delta_{i}}{2}, 0,0,0,0\right] \text {, and } \\
& \frac{\partial P_{i}}{\partial \Phi_{i+1}^{(n+1 / 2)}}=\left[\widetilde{\delta}_{i} \tau_{i, r} \hat{\beta}_{i, R}, 0,0,0,0,0\right], \\
& \frac{\partial N_{i}}{\partial \Phi_{i}^{(n+1 / 2)}}=\left[\left(\frac{\Delta_{i}}{2}+\widetilde{\delta}_{i} \tau_{i, r} \hat{\beta}_{i, L}\right), \hat{\alpha}_{i, R}, 0,0,0,0\right], \\
& \frac{\partial N_{i}}{\partial \Phi_{i-1}^{(n+1 / 2)}}=\left[0, \widetilde{\delta}_{i} \tau_{i, r} \hat{\beta}_{i, L}, 0,0,0,0\right] \text { and } \\
& \frac{\partial N_{i}}{\partial \Phi_{i+1}^{(n+1 / 2)}}=\left[\frac{\Delta_{i}}{2}, 0,0,0,0,0\right] .
\end{aligned}
$$

Finally, for the reconstruction of the Jacobian, we have to specify the second term of Eq. (71) which is characterized by the derivatives of the update operator $\widehat{P}$, expressed in Eq. 65. This term is composed by the following non-zero partial derivatives

$$
\begin{gathered}
\frac{\partial \widehat{P}_{i}}{\partial J_{i, L}^{+,(n+1 / 2)}}=\left[\begin{array}{cc}
-\frac{\psi_{L, 2}^{(n+1 / 2)}}{\left(J_{L}^{+,(n+1 / 2)}\right)^{2}} & 0 \\
0 & 0 \\
\frac{\hat{\beta}}{\left(J_{L}^{+}+J_{R}^{+}\right)^{(n+1 / 2)}} & \frac{\hat{\beta}}{\left(J_{L}^{+}+J_{R}^{+}\right)^{(n+1 / 2)}}
\end{array}\right], \\
\frac{\partial \widehat{P}_{i}}{\partial J_{i, R}^{+,(n+1 / 2)}}=\left[\begin{array}{cc}
0 & 0 \\
0 & -\frac{\psi_{R, 1}^{(n+1 / 2)}}{\left(J_{R}^{+,(n+1 / 2)}\right)^{2}} \\
\frac{\hat{\beta}}{\left(J_{L}^{+}+J_{R}^{+}\right)^{(n+1 / 2)}} & \frac{\hat{\beta}}{\left(J_{L}^{+}+J_{R}^{+}\right)^{(n+1 / 2)}}
\end{array}\right],
\end{gathered}
$$




$$
\frac{\partial \widehat{P}_{i}}{\partial \bar{\phi}_{i}^{(n+1 / 2)}}=\left[\begin{array}{cc}
0 & 0 \\
0 & 0 \\
\frac{1}{\left(J_{L}^{+}+J_{R}^{+}\right)^{(n+1 / 2)}} & \frac{1}{\left(J_{L}^{+}+J_{R}^{+}\right)^{(n+1 / 2)}}
\end{array}\right] .
$$

The Jacobian matrix (67) can be computed by reassembling Eq. (69) and (70) into Eq. (68). In particular, the derivatives contained in the matrix 70 are computed by Eqs. 72 and $(73)$ in 71 and by substituting all the derivatives appearing in 73 with Eqs. (74) to (98).

Note that the explicit computation of the Jacobian matrix allows the analysis of the convergence of the method far and close to the fixed point. Moreover, compared to the Fourier analysis tool when applied to non-linear methods, the study of the spectral radius of the Jacobian provides a numerical tool to analyze heterogeneous non-periodic geometries.

\subsection{Results spectral radius analysis}

Using the methodology defined in section 4 we perform a spectral radius analysis of SVRM and CMR using both the Gauss-Jacobi and Gauss-Seidel transport operator for the following case: finite 1D geometry composed of two computational cells with equivalent homogeneous, energy invariant, isotropic scattering cross section, and fixed sources, and periodic boundary conditions. The results of this analysis when $S_{2}$ or $S_{8}$ angular distributions are used, along with equivalent numerical benchmark results, are shown below in Figs 4.2 and 4.2 respectively. We can observe that the numerical results are in good agreement with the analytical results for all cases, validating the analysis we have done up until this point. An exact match between the numerical and analytical results is not expected do to the nonlinear nature of the SVRM operator and numerical in precision. For both the Gauss-Siedel and Gauss-Jacobi transport operators, the spectral radii of both versions of SVRM and CMR are identical. This result makes sense since we are solving a problem with flat flux solution, which means that the first order spatially variant equation, which differentiates SVRM from CMR has little effect. At large optical thickness we can observe that SVRM and CMR perform significantly better than transport since the spectral radius of transport is tied to the scattering ratio at large optical thicknesses [5]. 
Spectral Radius Analysis:
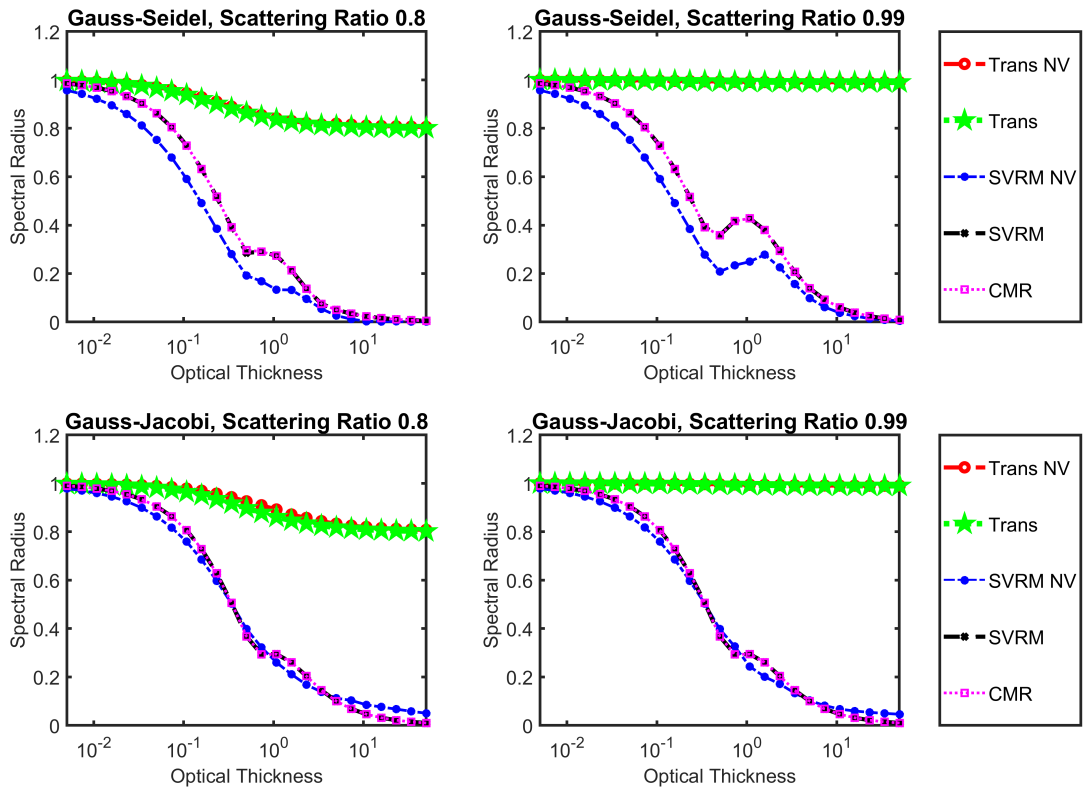

Figure 2: displays the spectral radius of SVRM for the problem defined in section 4.2 , when a $S_{2}$ angular distribution is used is used. NV stands for numerical validation. 
Spectral Radius Analysis:
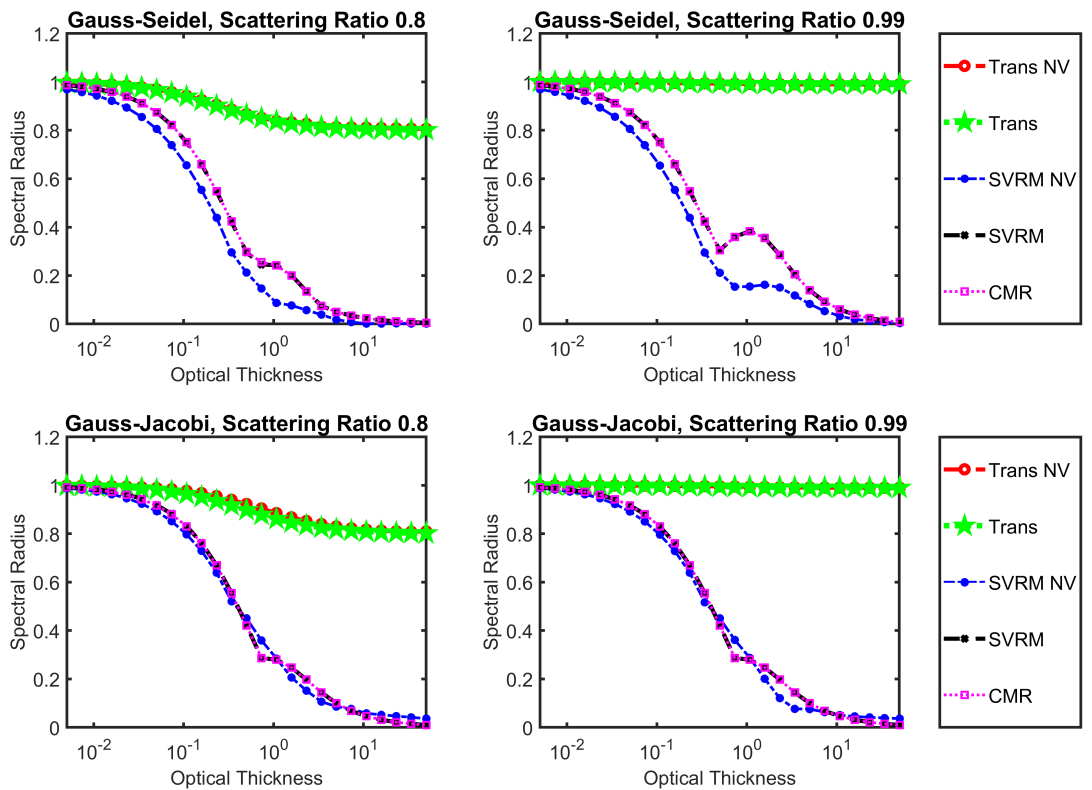

Figure 3: displays the spectral radius of SVRM for the problem defined in section 4.2 , when a $S_{8}$ angular distribution is used. NV stands for numerical validation.

\section{$5 \quad$ Fourier Analysis}

While the methodology in section 4 lets us compute the spectral radius for a generic problem, it is costly to compute for a problem with many cells. Thus, in order to look at the spectral radius of SVRM when the number of cells goes to infinite, we must use the following approach. Starting with Eq. (44), we focus on the constraints that define an interior spatial cell there are no boundary constraints. Because of the linearity of the transport operator, if we expand the iterative solution around the fixed point $\Lambda$ as

$$
\Psi_{i}^{(n+1 / 2)}=\Lambda+\partial \Psi_{i}^{(n+1 / 2)},
$$

where $\partial \Psi_{i}^{(n+1 / 2)}$ is the error and $\Lambda$ the solution verifying the equation $\Lambda=$ $\left(T_{i, i-1}+T_{i, i}+T_{i, i+1}\right) \Lambda+Q_{i}^{\prime}$, the transport contribution to the Jacobian is given by the transport matrix $T$. Following the Fourier ansatz for the principal component

$$
\partial \Psi_{i}^{(n+1 / 2)}=F_{i} \Xi^{(n+1 / 2)}
$$


where $\Xi$ is the error amplitudes

$$
\Xi^{(n+1 / 2)}=\left[\begin{array}{c}
\xi_{L} \\
\xi_{R} \\
\bar{\xi}
\end{array}\right]^{(n+1 / 2)}
$$

and $F_{i}$ is the Fourier matrix

$$
F_{i}=\left[\begin{array}{ccc}
e^{j \frac{2 \pi \zeta}{\Delta_{i}}\left(x_{i}-\frac{\Delta_{i}}{2}\right)} & 0 & 0 \\
0 & e^{j \frac{2 \pi \zeta}{\Delta_{i}}\left(x_{i}+\frac{\Delta_{i}}{2}\right)} & 0 \\
0 & 0 & e^{j \frac{2 \pi \zeta}{\Delta_{i}} x_{i}}
\end{array}\right],
$$

whit $x_{i}$ as the midpoint of region $i$, we can solve Eq. 44 for $\Xi^{(n+1 / 2)}$,

$$
\Xi^{(n+1 / 2)}=F_{i, i}^{-1}\left(T_{i, i-1} F_{i, i-1}+T_{i, i} F_{i, i}+T_{i, i+1} F_{i, i+1}\right) \Xi^{(n)}
$$

We do the same expansion around the fixed point for the partial currents $J_{i}^{(n+1)}$ as

$$
J_{i}^{(n+1)}=\frac{\Lambda}{4}+\partial J_{i}^{(n+1)}
$$

where the error $\partial J_{i}^{(n+1)}$ is expanded on the principal Fourier mode $\Theta$ as

$$
\partial J_{i}^{(n+1)}=\widetilde{F}_{i} \Theta^{(n+1)},
$$

where

$$
\Theta^{(n+1)}=\left[\begin{array}{l}
\theta_{L} \\
\theta_{R}
\end{array}\right]^{(n+1)}
$$

and

$$
\widetilde{F}_{i}=\left[\begin{array}{cc}
e^{j \frac{2 \pi \zeta}{\Delta_{i}}\left(x_{i}-\frac{\Delta_{i}}{2}\right)} & 0 \\
0 & e^{j \frac{2 \pi \zeta}{\Delta_{i}}\left(x_{i}+\frac{\Delta_{i}}{2}\right)}
\end{array}\right] .
$$

By specializing the prolongation equation (64) for region $i$, the updated error variation is given by a direct derivation of (64), which gives

$$
\begin{aligned}
\partial \Psi_{i}^{(n+1)} & =\widehat{P}_{i, i} \partial J_{i}^{(n+1)}+\left.\sum_{j \in(i-1, i, i+1)} \frac{\partial\left(\widehat{P}_{i, i} J_{i}^{(n+1)}\right)}{\partial \Phi_{j}^{(n+1 / 2)}}\right|_{\Phi_{j}^{(n+1 / 2)}=K_{j} \Lambda} \partial \Phi_{j}^{\left(n+1 \mathbb{1}^{1} \partial \mathrm{I}^{2}\right)} \\
& \simeq \widehat{P}_{i, i} \partial J_{i}^{(n+1)}+\left.\frac{\Lambda}{4} \sum_{j \in(i-1, i, i+1)} \frac{\partial\left(\widehat{P}_{i, i} 1\right)}{\partial \Phi_{j}^{(n+1 / 2)}}\right|_{\Phi_{j}^{(n+1 / 2)}=K_{j} \Lambda} \partial \Phi_{j}^{(n+1 / 2)}
\end{aligned}
$$

The first term on the rhs of Eq. (101) can be obtained by Eq. (73), in fact, the error on the partial current $\partial J_{i}^{(n+1)}$ is computed by differentiating 73 as $\hat{A}_{i, i-1} \partial J_{i-1}^{(n+1)}+\hat{A}_{i, i} \partial J_{i}^{(n+1)}+\hat{A}_{i, i+1} \partial J_{i+1}^{(n+1)}=-\left(\partial \hat{A}_{i, i-1} J_{i-1}^{(n+1)}+\partial \hat{A}_{i, i} J_{i}^{(n+1)}+\partial \hat{A}_{i, i+1} J_{i+1}^{(n+1)}\right)$. 
Substituting the Fourier ansatz 100 for the partial currents in previous equa-

tion and specializing the equation near the convergence, i.e. $J_{L / R}^{+}=\frac{\Lambda}{4}$, we obtain

$$
\begin{aligned}
\Theta^{(n+1)} & =\frac{\Lambda}{4}\left(\sum_{j \in(i-1, i, i+1)} \hat{A}_{i, j} \widetilde{F}_{j}\right)^{-1} \sum_{j \in(i-1, i, i+1)} \partial \hat{A}_{i, j} \mathbf{1} \\
& =\left[\frac{\Lambda}{4}\left(\sum_{j \in(i-1, i, i+1)} \hat{A}_{i, j} \widetilde{F}_{j}\right)_{j \in(i-1, i, i+1)} \hat{H}_{i, j}\right] \Xi^{(n+1 / 2)} .
\end{aligned}
$$

In 102$), \partial \hat{A}_{i, j} \mathbf{1}$ is the vector summing all the columns of matrix $\partial \hat{A}_{i, j}$ ( $\mathbf{1}$ here is the unitary vector of dimension 2 ). After algebraic manipulations, the vector $\partial \hat{A}_{i, j} \mathbf{1}$ can be expressed by

$$
\partial \hat{A}_{i, j} \mathbf{1}=\widehat{H}_{i, j} \Xi^{(n+1 / 2)}
$$

where the differential matrix $\widehat{H}_{i, j}$ is

$$
\widehat{H}_{i, j}=\left.\sum_{x \in\left(\hat{\alpha}_{i, R}, \hat{\alpha}_{i, L}, \hat{\beta}_{i, R}, \hat{\beta}_{i, L}, \hat{\beta}_{i}, \widehat{\eta}_{i}\right)} \frac{\partial \hat{A}_{i, j}}{\partial x} \mathbf{1} \otimes \sum_{k \in(i-1, i, i+1)} \frac{\partial x}{\partial \Phi_{k}^{(n+1 / 2)}}\right|_{\Phi_{k}^{(n+1 / 2)}=K_{k} \Lambda} K_{k, k} F_{k} .
$$

The matrices $\widehat{H}_{i, j}$ are computed using the non zero derivatives 74 - $(95)$. Then, using the Fourier ansatz in Eq. 101) and substituting 102 for $\Theta^{(n+1)}$, we obtain the final definition for the iterative matrix $\widehat{G}$

$$
\begin{aligned}
\Xi^{(n+1)}= & \frac{\Lambda}{4} F_{i}^{-1}\left\{\widehat{P}_{i, i} \widetilde{F}_{i}\left(\sum_{j \in(i-1, i, i+1)} \hat{A}_{i, j} \widetilde{F}_{j}\right)_{j \in(i-1, i, i+1)}^{-1} \widehat{H}_{i, j}(104)\right. \\
& \left.\left.\sum_{j \in(i-1, i, i+1)} \frac{\partial\left(\widehat{P}_{i, i} \mathbf{1}\right)}{\partial \Phi_{j}^{(n+1 / 2)}}\right|_{\Lambda} K_{j} F_{j}\right\} \Xi^{(n+1 / 2)} \\
= & \widehat{G} \Xi^{(n)}
\end{aligned}
$$

where the derivatives $\frac{\partial\left(\widehat{P}_{i, i} \mathbf{1}\right)}{\partial \Phi_{j}^{(n+1 / 2)}}$ are computed thanks to $96-(98$. We analyze the spectral radius of the matrix $\widehat{G}$ given by

$$
\begin{aligned}
\widehat{G}= & \frac{\Lambda}{4}\left\{\left.F_{i}^{-1} \widehat{P}_{i, i}\right|_{\Lambda} \widetilde{F}_{i}\left(\left.\sum_{j \in(i-1, i, i+1)} \hat{A}_{i, j}\right|_{\Lambda} \widetilde{F}_{j}\right)^{-1} \sum_{j \in(i-1, i, i+1)} \widehat{H}_{i, \lambda}(105)\right. \\
& \left.\left.\sum_{j \in(i-1, i, i+1)} \frac{\partial\left(\widehat{\widehat{P}}_{i, i} 1\right)}{\partial \Phi_{j}^{(n+1 / 2)}}\right|_{\Lambda} K_{j, j} F_{j}\right\} K_{i, i} F_{i, i}^{-1} \sum_{j \in(i-1, i, i+1)} T_{i, j} F_{i, j}
\end{aligned}
$$


where use have been made of $(99)$. All the matrices and derivatives 74 to 95 are computed at the fixed point by considering the following identities

$$
\Psi_{i} \equiv \Phi_{i} \equiv 4 J_{i} \equiv \Lambda
$$

and, consequently,

$$
\begin{aligned}
\hat{\alpha}_{i, R} & =\hat{\alpha}_{i, L}=2 w|\mu| b, \\
\hat{\beta}_{i, R} & =\hat{\beta}_{i, L}=\hat{\beta}_{i}=2, \\
\widehat{\eta}_{i} & =1 / 2 .
\end{aligned}
$$

\subsection{Results of Fourier analysis}

Using the methodology defined in section 5 we achieve the results shown by Fig. 5.1. By comparing the results of the Fourier analysis with those of the spectral radius analysis, we can observe that that when the spatial mesh is increased from two cells to an infinite grid, the performance of SVRM is relatively unaffected. This is a good indication the performance of SVRM will hold for larger scale problems then what have been tested in this article.

\section{Fourier Analysis}
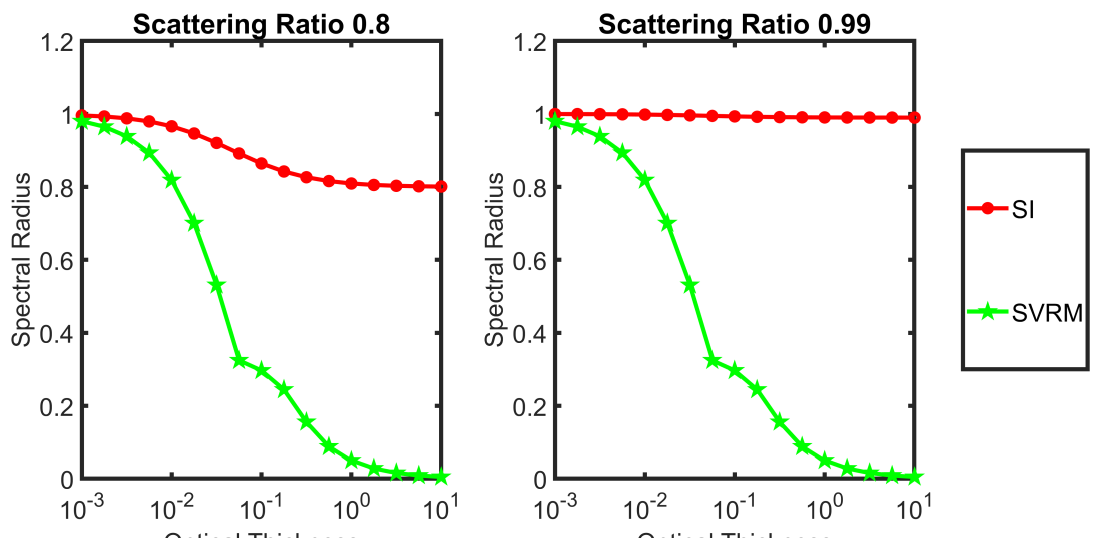

Figure 4: displays the spectral radius of SVRM for the infinite spatial mesh problem defined in section 5, when the piece-wise and the quadratic spatial distributions are used.

\section{Numerical Benchmark}

The accuracy of equation relating the surface current to the scalar flux significantly affects the performance and stability of the method. The most dramatic changes in the flux and current profile occur at boundaries with large material 
discontinuities. Subsequently the effect of the accuracy of the scalar flux and surface current relationship is of greatest significance at these highly discontinuous boundaries. Thus, in order to investigate the effects the scalar flux and surface current relationship used by SVRM, have on it's performance, we must study the behaviour of SVRM at boundaries with large material discontinuities. In order to study a wide range of discontinuities in a time efficient manor, we chose to use the simple benchmark case described below.

The benchmark problem has: one energy group, two cells, a fixed isotropic source, $S_{8}$ angular distribution, and reflective boundary conditions. To explore a wide range of material discontinuities the total cross-section and the scattering cross-section are fixed for one cell and varied for the other according to the following equation.

$$
\tau_{2}(P)=\frac{\left(1-c_{1}\right) \tau_{1}}{P}+P c_{1} \tau_{1} \quad c_{2}(P)=\frac{P c_{1} \tau_{1}}{\frac{\left(1-c_{1}\right) \tau_{1}}{P}+P c_{1} \tau_{1}}
$$

A visualization summarizing the relation between $P$ and $\tau_{2}$ is shown in Fig. 6. The total cross section of material 2 of Eq. (106) is such that the material 2 becomes an infinite absorber as $P$ goes to zero while it becomes an infinite diffusive material as $P$ increases to the infinite.

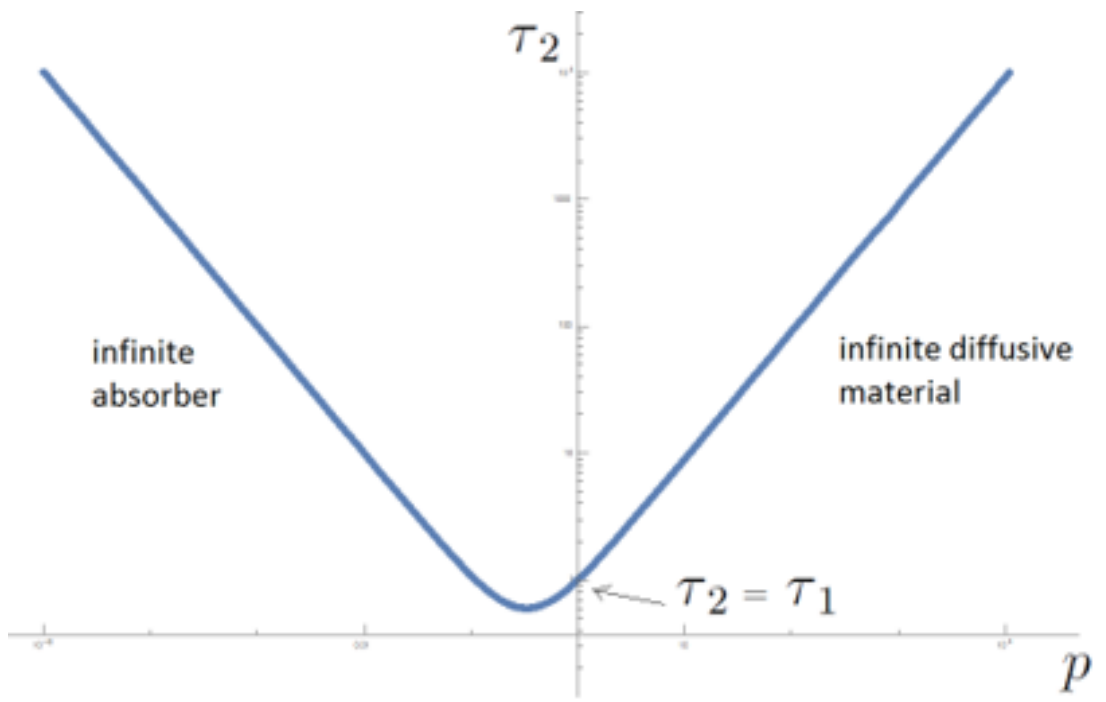

(B)

Figure 5: shows how $\tau_{2}$ varies as a function of $P$, according to Eq 106

The cell with fixed properties was studied for three different $\tau_{1}$ values $(0.005,0.5,50)$, 
and for three different $c_{1}$ values $(0.5,0.9,0.99)$, for a total of nine variants. The SVRM has been compared to the acceleration methods already available in the 1D slab solver, in particular, to the DSA and the BPA. Plots showing how the number of iterations needed for convergence varies with $P$, for the unaccelerated transport, SVRM, DSA, and BPA operators, and for each of these nine cases are shown section 6.1 .

\subsection{Results of numerical benchmark}

Using the methodology described in section 6 we produce the results displayed by Figs 6.1 to 6.1. Starting with Fig 6.1 below, SVRM can be seen to converge $10 \rightarrow 1000$ times faster than DSA, BPA, CMFD and transport for all values of $P$. The relative performance advantage of SVRM can be seen to increase as the variable medium becomes more diffusive (highier $P$ value).
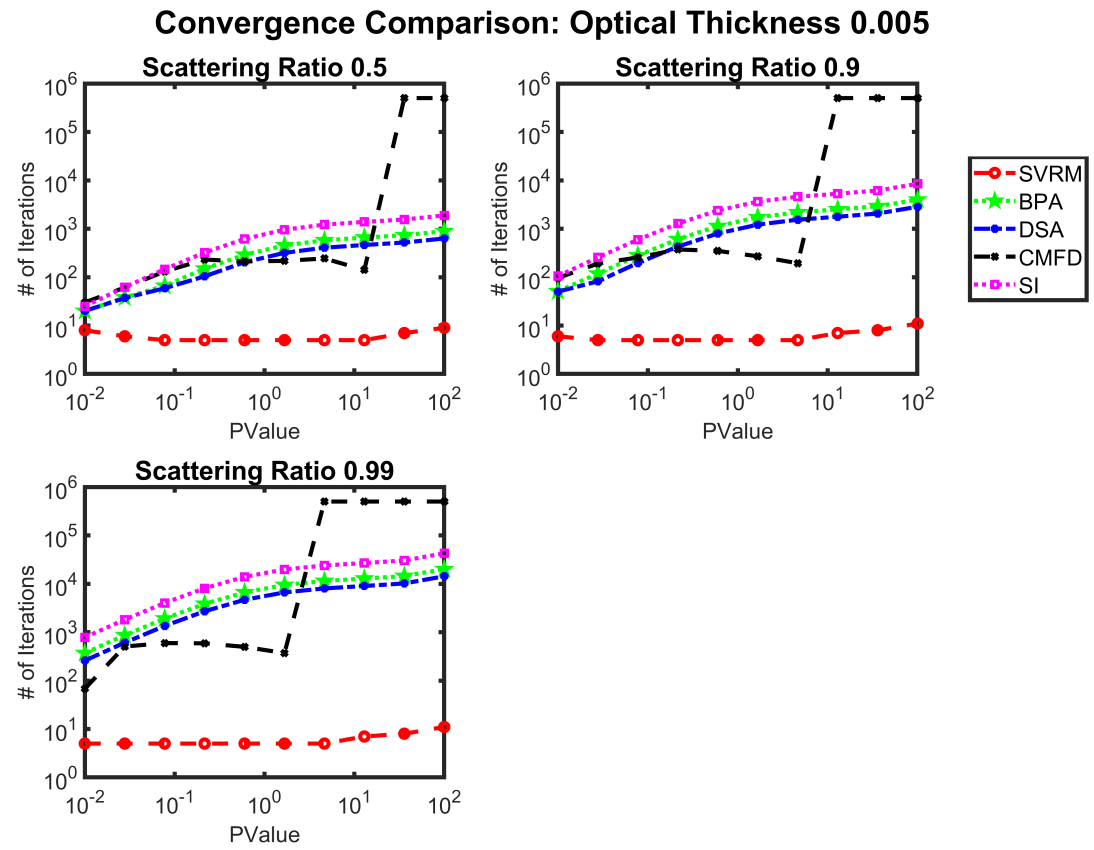

Figure 6: Benchmarks the performance (number iterations required to reach a converged solution) of SVRM against DSA, BPA, CMFD, and SI using the methodology described in section 6 when the fixed cell has an optical thickness of 0.005 . Iteration values of $5 \times 10^{5}$ indicate instability.

From Fig 6.1 below, we can observe that when the optical thickness of the 
fixed cell is increased to a value of 0.5, the performance advantage of SVRM has degraded, relative to the other methods. However SVRM can still be seen to provide a significant performance advantage over all other methods. DSA, $\mathrm{BPA}$, and transport continue to follow the the same trend as before, with the number of iterations required for them to converge increasing as the variable cell is made more diffusive. For small values of $P$ we can see that DSA is unstable. CMFD can be observed to provide good performance until it becomes unstable at large values of $P$.
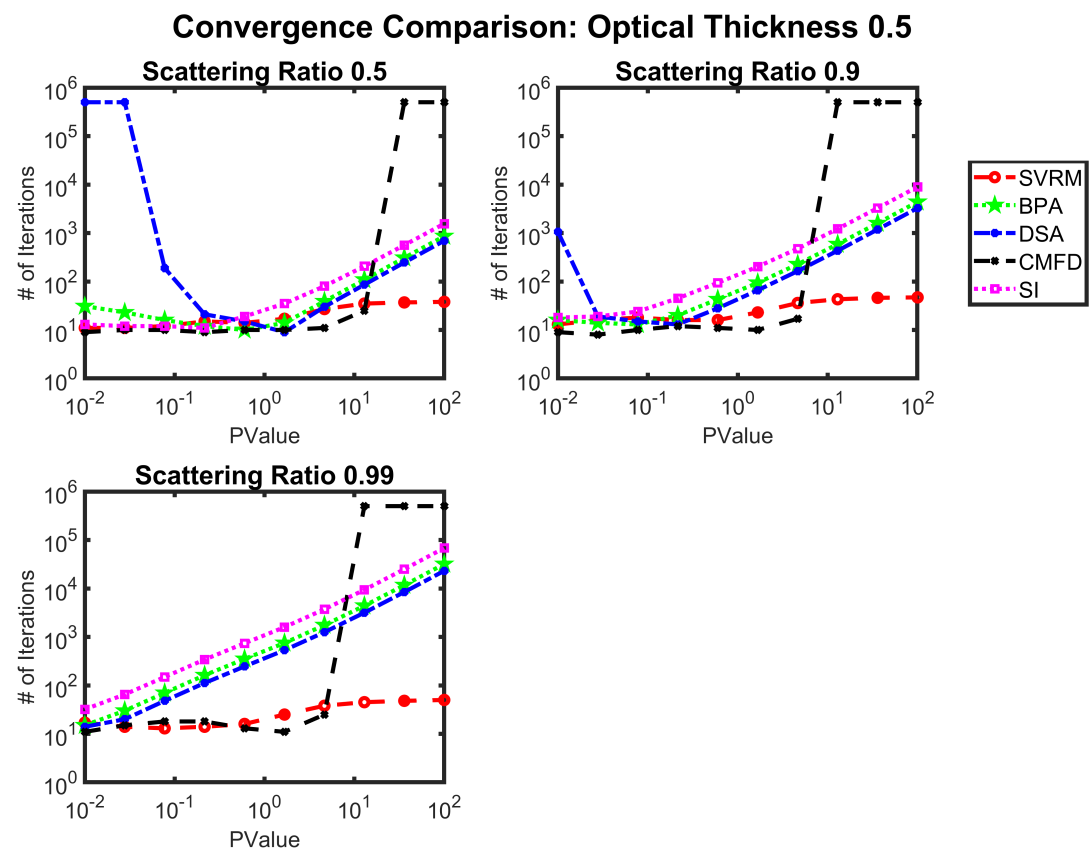

Figure 7: Benchmarks the performance (number iterations required to reach a converged solution) of SVRM against DSA, BPA, CMFD, and SI using the methodology described in section 6 when the fixed cell has an optical thickness of 0.5 . Iteration values of $5 \times 10^{5}$ indicate instability.

In Fig 6.1 below we can observe that when we increase the optical thickness of the fixed cell to a value of 50, the relative performance of SVRM method continues to degrade. While SVRM still retains a performance advantage over DSA and BPA, it is now significantly less effective than CMFD. 

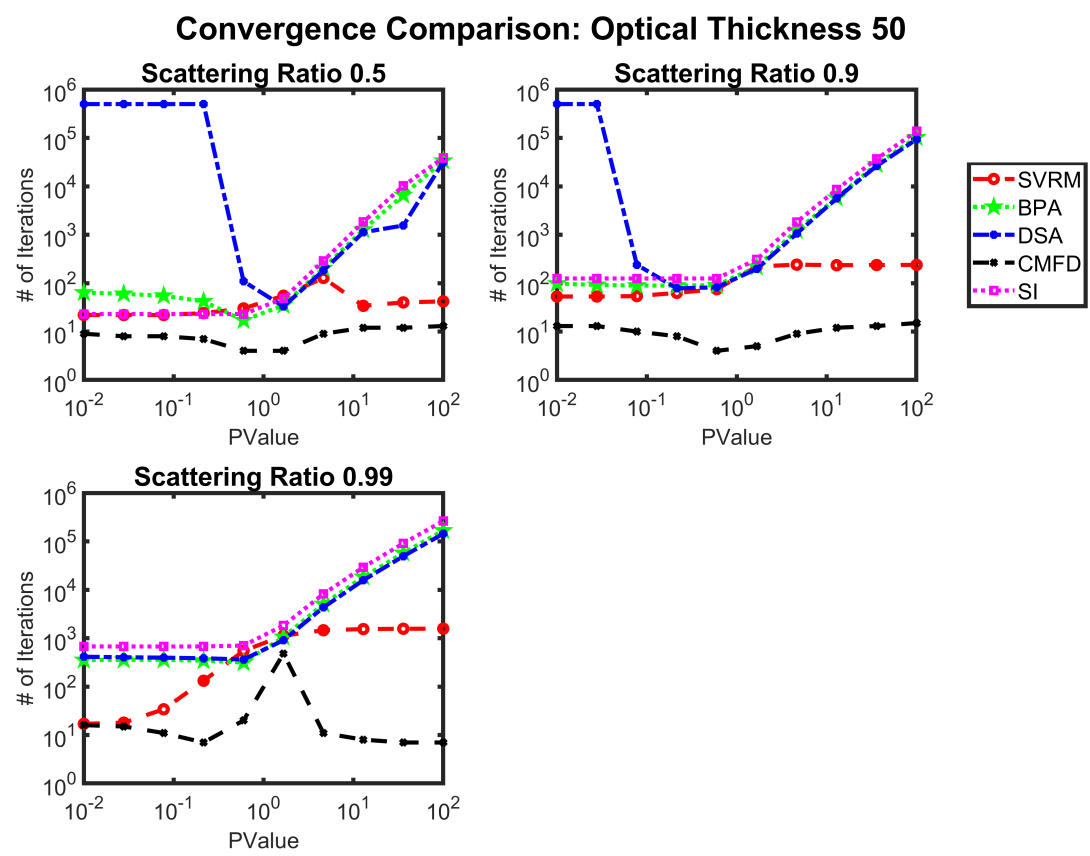

Figure 8: Benchmarks the performance (number iterations required to reach a converged solution) of SVRM against DSA, BPA, CMFD, and SI using the methodology described in section 6 when the fixed cell has an optical thickness of 50 . Iteration values of $5 \times 10^{5}$ indicate instability.

\section{Conclusion and further remarks}

In this paper we propose a new class of rebalancing methods. SVRM opens up a new frontier for numerical research in non-linear accelerations. As was previously explained, SVRM is based on linear variation of the particle balance equation. This aspect is an important improvement for the effectiveness and for the stability of the acceleration, as demonstrated by the spectral analysis and the Fourier analysis and the numerical benchmarks. Further refinement of the SVRM by way of improving the closure relations, is already underway and is expected to greatly improve the relative performance of the method (particular with respect to CMFD).

Compared to other rebalancing method, SVRM doubles the numbers of unknowns. However, in this paper we have limited our analysis to a particular case when transport and the acceleration shares the same spatial mesh. Further investigation will be done by applying the SVRM operator to coarse meshes. This 
field will be important to find the compromise among the memory burden and the effectiveness of the acceleration. We have also limited the application of the SVRM to the inner iterations, but the method could be easily applied to multigroup eigenvalue problems as a preconditioner for power and thermal iterations.

Another aspect earning further investigation is the application of SVRM to high-order MOC. For example, the linear MOC satisfies automatically the first-order balance equation, therefore the exact first order variation of the flux and of the source will be available for use by SVRM. In this case, the SVRM will be automatically consistent with the transport and, as a consequence, the consistency parameter $\widehat{\eta}$ can be eliminated.

An important improvement can also be achieved by using finite differences relation for the closures relations. This particular research filed open the doors to a variety of class of spatially variant non-linear methods. Finally, we conclude, that a multidimensional version of the SVRM method is under development in our laboratory for application to real-scale reactor calculation.

\section{References}

[1] Marvin L. Adams and Edward W. Larsen. Fast iterative methods for discrete-ordinates particle transport calculations. Progress in Nuclear Energy, 40(1):3-159, 2002.

[2] Gregory R. Cefus and Edward W. Larsen. Stability analysis of coarse-mesh rebalance. Nuclear Science and Engineering, 105(1):31-39, 1990.

[3] Hyeon Tae Kim and Yonghee Kim. Convergence studies on nonlinear coarsemesh finite difference accelerations for neutron transport analysis. Nuclear Science and Engineering, 191(2):136-149, 2018.

[4] N.Z. Cho and C.J. Park. A comparison of coarse mesh rebalance and coarse mesh finite difference accelerations for the neutron transport calculations. Nuclear Mathematical and Computational Sciences: A Century in Review, 2003.

[5] J.R. Askew. A characteristics formulation of the neutron transport equation in complicated geometries. INIS, 1972. 